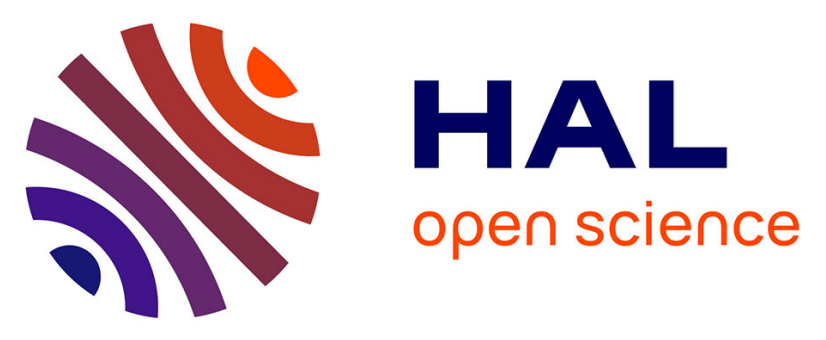

\title{
Actualisation des Recommandations de la Société française de rhumatologie pour la prise en charge de la polyarthrite rhumatoïde
}

Claire Daien, Charlotte Hua, Cécile Gaujoux-Viala, Alain Cantagrel, Madeleine Dubremetz, Maxime Dougados, Bruno Fautrel, Xavier Mariette, Nathalie Nayral, Christophe Richez, et al.

\section{To cite this version:}

Claire Daien, Charlotte Hua, Cécile Gaujoux-Viala, Alain Cantagrel, Madeleine Dubremetz, et al.. Actualisation des Recommandations de la Société française de rhumatologie pour la prise en charge de la polyarthrite rhumatoïde. Revue du Rhumatisme, 2019, 86, pp.8 - 24. 10.1016/j.rhum.2018.09.008 . hal-03486207

\section{HAL Id: hal-03486207 \\ https://hal.science/hal-03486207}

Submitted on 20 Dec 2021

HAL is a multi-disciplinary open access archive for the deposit and dissemination of scientific research documents, whether they are published or not. The documents may come from teaching and research institutions in France or abroad, or from public or private research centers.
L'archive ouverte pluridisciplinaire HAL, est destinée au dépôt et à la diffusion de documents scientifiques de niveau recherche, publiés ou non, émanant des établissements d'enseignement et de recherche français ou étrangers, des laboratoires publics ou privés.

\section{다)(1) $\$$}

Distributed under a Creative Commons Attribution - NonCommerciall 4.0 International 


\section{Actualisation des Recommandations de la Société française de rhumatologie pour la prise en charge de la polyarthrite rhumatoïde}

Claire Daien ${ }^{\mathrm{a}, \mathrm{b}} *$, Charlotte Hua ${ }^{\mathrm{c}, \mathrm{d} *}$, Cécile Gaujoux-Viala ${ }^{\mathrm{c}, \mathrm{d}}$, Alain Cantagrel ${ }^{\mathrm{e}, \mathrm{f},}$ Madeleine Dubremetz $^{\mathrm{g}}$, Maxime Dougados ${ }^{\mathrm{h}, \mathrm{i}, \mathrm{j}}$, Bruno Fautrel ${ }^{\mathrm{k}, \mathrm{l}}$, Xavier Mariette ${ }^{\mathrm{m}, \mathrm{n},}$ Nathalie Nayral ${ }^{\mathrm{a}, \mathrm{b}}$, Christophe Richez $^{\mathrm{o}, \mathrm{p}}$, Alain Saraux ${ }^{\mathrm{q}, \mathrm{r}}$, Gérard Thibaud ${ }^{\mathrm{s}}$, Daniel Wendling ${ }^{\mathrm{t}, \mathrm{u}}$, Laure Gossec ${ }^{\mathrm{k}, 1_{*}}$, Bernard Combe $e^{\mathrm{a}, \mathrm{b} *}$

${ }^{\text {a }}$ Service de Rhumatologie, CHU de Montpellier, 34295 Montpellier, France

${ }^{\mathrm{b}}$ Université de Montpellier, 34295 Montpellier, France

${ }^{\text {c,d }}$ Service de Rhumatologie, CHU de Nîmes, 30029 Nîmes, France

EA2415, Université de Montpellier, 30029 Nîmes, France

${ }^{\mathrm{e}}$ Service de Rhumatologie, CHU de Toulouse, 31059 Toulouse, France

${ }^{\mathrm{f}}$ Université Toulouse III, UMR Inserm 1043-CNRS 5282, 31059 Toulouse, France

${ }^{g}$ Association française des Polyarthritiques et des Rhumatismes Inflammatoires Chroniques (AFP RIC), 75011 Paris, France

${ }^{\mathrm{h}}$ Paris Descartes University, 75006 Paris, France

${ }^{\mathrm{i}}$ Department of Rheumatology - Hôpital Cochin. Assistance Publique - Hôpitaux de Paris, 75006 Paris, France

${ }^{\mathrm{j}}$ INSERM (U1153): Clinical epidemiology and biostatistics, PRES Sorbonne Paris-Cité.

75006 Paris, France

${ }^{\mathrm{k}}$ Sorbonne Université, 75013 Paris France;

${ }^{1}$ Pitié Salpêtrière hospital, APHP, Rheumatology department, 75013 Paris, France

${ }^{\mathrm{m}}$ Service de Rhumatologie, Hôpitaux Universitaires Paris-Sud, AP-HP, 94270 Le KremlinBicêtre, France

${ }^{\mathrm{n}}$ Université Paris-Sud, INSERM UMR1184, 94270 Le Kremlin-Bicêtre, France

${ }^{\mathrm{o}}$ Service de Rhumatologie, CHU de Bordeaux, 33000 Bordeaux, France

${ }^{\mathrm{p}}$ Université de Bordeaux, 33000 Bordeaux, France

${ }^{\mathrm{q}}$ Service de Rhumatologie, CHU de Brest, 29200 Brest, France

${ }^{\mathrm{r}}$ INSERM 1227, Université de Bretagne occidentale, 29200 Brest, France 


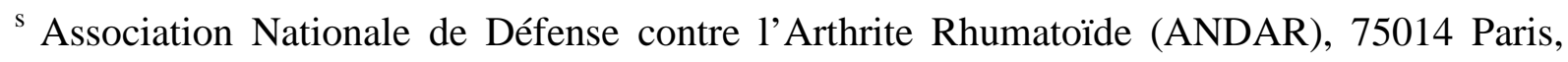
France

${ }^{\mathrm{t}}$ Service de Rhumatologie, CHU de Besançon, 25000 Besançon, France

${ }^{\mathrm{u} U n i v e r s i t e ́ ~ d e ~ F r a n c h e-C o m t e ́, ~} 25000$ Besançon, France

Auteur Correspondant: Dr Claire Daien, Département de Rhumatologie, Hôpital Lapeyronie, 371 av du Doyen Giraud, 34295 Montpellier Montpellier, France ·

c-daien@chu-montpellier.fr

* $\mathrm{CD}$ et $\mathrm{CH}$ co-premier auteurs, $\mathrm{CD}$ et $\mathrm{CH}$ ont contribué de manière équivalente. $\mathrm{LG}$ et $\mathrm{BC}$ co-dernier auteurs, LG et $\mathrm{BC}$ ont contribué de manière équivalente. 


\section{RESUME}

Les précédentes recommandations de 2014 ont été actualisées par un groupe de travail constitué de 12 experts rhumatologues, deux représentants des associations de patients et un professionnel de santé. Le groupe de travail s'est appuyé sur les recommandations de l'EULAR récemment publiées, sur une analyse systématique de la littérature et sur l'avis des experts. Quatre principes généraux et 15 recommandations ont été émis. Les principes généraux mettent en avant l'importance d'une décision partagée entre le rhumatologue et le patient et la nécessité d'une prise en charge globale de la polyarthrite rhumatoïde (PR), sur le plan médicamenteux et non-médicamenteux. Les recommandations concernent l'approche diagnostique de la PR, les objectifs du traitement, l'organisation de la prise en charge, les traitements médicamenteux à utiliser en fonction de la ligne de traitement et des facteurs pronostiques, la gestion de la rémission et la prise en charge globale des patients. Le traitement de fond (Disease Modifying Anti-Rheumatic Drug [DMARD]) doit être initié le plus précocement possible. L'évaluation de l'activité de la maladie par des scores composites validés doit être régulière (contrôle serré) pour atteindre l'objectif thérapeutique (la rémission). Le méthotrexate (MTX) est recommandé en première intention. En cas d'intolérance ou de réponse insuffisante au MTX, le traitement doit être optimisé. En attendant que les DMARDs synthétiques conventionnels soient efficaces, une corticothérapie peut être proposée sur une courte durée afin de garder une dose cumulée faible. En cas de rémission persistante, sans progression structurale, sans corticoïdes, une décroissance progressive des thérapeutiques ciblées respectant les principes du contrôle serré doit être envisagée. Un dépistage et une évaluation périodique des comorbidités, de leurs facteurs de risque et de leur prise en charge doivent être réalisés.

Mots clés : polyarthrite rhumatoïde ; diagnostic ; traitement ; traitement ciblé ; DMARD ; recommandations 


\section{Introduction}

Depuis les dernières recommandations françaises pour la prise en charge de la polyarthrite rhumatoïde (PR) élaborées en 2014 [1], de nouvelles données ont été publiées. Ces données concernent notamment les traitements de la PR (avec la mise sur le marché de nouveaux médicaments et l'évaluation de stratégies thérapeutiques, notamment en cas de rémission), la prise en charge non médicamenteuse (rééducation et éducation thérapeutique), et la gestion des comorbidités associées. La mise à jour régulière des recommandations, basée sur les données actualisées de la littérature, est nécessaire pour guider au mieux les différents acteurs impliqués dans la prise en charge de cette pathologie et continuer à améliorer la qualité de vie, le pronostic fonctionnel et le pronostic vital des patients atteints de PR. L'European League Against Rheumatisms (EULAR) actualise également régulièrement ses recommandations et a publié en 2016 la mise à jour des recommandations concernant la PR et les arthrites récentes $[2,3]$.

Les recommandations françaises ont pour objectif de couvrir tous les domaines de la prise en charge de la PR : diagnostic, traitement, suivi, gestion de la rémission, prise en charge des comorbidités. Elles sont destinées en premier lieu aux rhumatologues mais peuvent aussi être utiles aux autres médecins prenant en charge les patients atteints de PR, notamment les médecins généralistes, aux autres professionnels de santé, aux étudiants, aux associations de patients et aux autorités de santé.

L'objectif de ce travail était donc d'actualiser les recommandations de la société française de rhumatologie (SFR) pour la prise en charge de la PR afin d'améliorer la prise en charge globale des patients atteints de PR. 


\section{Méthodes}

Un groupe de travail désigné par la société française de rhumatologie (SFR), constitué de 12 rhumatologues hospitaliers ou libéraux, deux patients atteints de PR et membres des associations de patients et une professionnelle de santé (ergothérapeute), exerçant dans toute la France, s'est réuni pour élaborer ces recommandations. Le groupe de travail s'est appuyé sur les précédentes recommandations de la SFR (2014) et de l'EULAR (2016) pour la prise en charge de la PR et des arthrites récentes [1-3]. De plus, une revue systématique de la littérature a été réalisée (C.D et C.H) afin de recueillir les données publiées depuis les dates de fin des dernières analyses de la littérature réalisées pour établir les précédentes recommandations (entre novembre 2015 et février 2016 selon les items) et jusqu'en octobre 2017 [4-8]. Cette revue systématique de la littérature a été conduite à partir de la base de données MedLine (via PubMed) et à partir des abstracts publiés aux congrès 2016 et 2017 de l'EULAR et de l'American College of Rheumatology (ACR). L'objectif de cette revue systématique de la littérature était d'identifier les données récentes publiées afin de répondre à 11 questions préétablies par le groupe de travail. Ces questions portaient sur les items suivants : délai de prise en charge de la PR et fenêtre d'opportunité, diagnostic, pronostic, traitements et stratégies thérapeutiques, gestion de la rémission, prise en charge globale et des comorbidités. L'actualisation des recommandations françaises a été élaborée par le groupe de travail en janvier 2018, puis finalisée après plusieurs tours de courriers électroniques. Les recommandations émises ont ensuite été relues par un groupe de lecture constitué de 40 experts, rhumatologues hospitaliers et libéraux, professionnels de santé et patients désignés par les associations de patients. Les membres de ce groupe de lecture ont voté sur une échelle de 0 à 10 pour chaque recommandation : une note de 0 correspondait à un désaccord total avec la recommandation tandis qu'une note de 10 indiquait un accord complet. Les 
commentaires ont été pris en compte dans le libellé de certaines recommandations et dans ce texte. Les niveaux de preuve et grades des recommandations ont été déterminés [9].

\section{Résultats}

Le Tableau 1 définit les principaux termes utilisés et la figure 1 précise la nomenclature des traitements de fond. Les recommandations sont composées de 4 principes généraux et 15 recommandations (Tableau 2). Les niveaux de preuve, grades des recommandations et taux d'accord avec chaque recommandation du groupe de travail et du groupe de lecture sont aussi présentés dans le Tableau 2.

\subsection{Principes généraux}

Principe A : La prise en charge des patients atteints de PR nécessite une concertation entre le rhumatologue et le patient, dans le cadre d'une décision médicale partagée reposant sur l'information et l'éducation du patient.

Ce principe n'a pas été modifié par rapport à 2014. La PR étant une maladie chronique, le patient doit être impliqué dans sa prise en charge. «La décision médicale partagée », véritable socle de l'alliance thérapeutique, nécessite que tout patient puisse accéder à une information et un processus d'éducation qui lui permettent d'acquérir des connaissances importantes sur la maladie et sa gestion, et de participer à une prise de décision éclairée en concertation avec son rhumatologue [1,10,11]. Le diagnostic de PR devrait être annoncé au patient par le rhumatologue au cours d'une consultation d'annonce dédiée au cours de laquelle le projet thérapeutique sera élaboré avec le patient. L'éducation thérapeutique favorise l'autonomisation (notion d'auto-efficacité ou self-efficacy) et l'émergence du concept de 
patient-partenaire; elle va de ce fait au-delà de l'éducation sur les traitements médicamenteux. Selon la HAS, une éducation thérapeutique initiale doit être proposée au patient à un moment proche de l'annonce du diagnostic, ou à tout autre moment si la proposition ne lui a pas été faite antérieurement ou s'il l'a refusée [12]. Pour l'EULAR, tous les patients atteints de rhumatismes inflammatoires chroniques devraient avoir accès à une éducation thérapeutique au cours de leur maladie, y compris et au minimum, au moment du diagnostic (phase d'annonce), lors d'un changement de traitement médicamenteux (phase d'amorce) et lorsque leur état physique ou psychologique le nécessite [11]. La HAS recommande d'élaborer une démarche d'éducation thérapeutique standardisée en 4 étapes : l'élaboration d'un diagnostic éducatif, la définition d'un programme personnalisé d'éducation thérapeutique (intégrant une priorisation des objectifs), la mise en œuvre des séances d'éducation thérapeutique qui peuvent être individuelles ou collectives, l'évaluation des compétences acquises [12]. L'EULAR donne une définition plus large des modalités des programmes d'éducation thérapeutique pouvant être proposés aux patients [11]. Le groupe d'experts de la SFR, conscient du fait que les possibilités de mise en place des programmes et d'accès à l'éducation thérapeutique ne sont pas identiques sur l'ensemble du territoire français, considère que la démarche d'éducation thérapeutique doit être envisagée pour chaque patient mais que sa réalisation peut se faire sous différentes modalités en fonction des possibilités locales.

Principe B : Le rhumatologue est le spécialiste qui doit prendre en charge les patients atteints de PR. Le médecin généraliste joue un rôle important pour la détection de la maladie et, en coordination avec le rhumatologue, pour le suivi du patient. 
Ce principe n'a pas été modifié par rapport à 2014. La notion de prise en charge de la PR par les rhumatologues fait aussi partie des recommandations récentes de l'EULAR [2,3]. Ce principe se base notamment sur les données de la littérature indiquant que les patients atteints de PR bénéficient d'un diagnostic et d'un traitement plus précoces lorsqu'ils sont pris en charge par un médecin spécialiste rhumatologue, ce qui améliore ainsi leur pronostic fonctionnel et structural [4]. Cependant, le médecin généraliste est en première ligne pour détecter une possible PR débutante [13] et adresser très rapidement le patient au rhumatologue. Le rôle du médecin généraliste est également primordial pour le suivi du patient, de ses traitements et de la prise en charge des comorbidités. Le médecin généraliste peut aussi parfois être impliqué, en partenariat avec le rhumatologue, dans les renouvellements de traitements et les ajustements thérapeutiques. Le rhumatologue et le médecin généraliste participent donc conjointement au parcours de soins des patients atteints de PR, permettant ainsi un accompagnement de ces patients à la fois personnalisé et coordonné.

Principe C: Tout patient atteint de PR devrait bénéficier d'une prise en charge globale incluant les traitements médicamenteux, des mesures d'éducation thérapeutique et, selon les cas, une prise en charge des comorbidités, un soutien psychologique, un accompagnement socioprofessionnel, une rééducation fonctionnelle ou le recours à la chirurgie.

Il s'agit d'un nouveau principe qui correspond à la quinzième recommandation de 2014. Le groupe d'expert a considéré que cette affirmation générique représente un principe général plutôt qu'une recommandation. En effet, une prise en charge globale, comportant les prises en charge médicamenteuses et non médicamenteuses, devrait être proposée et adaptée à chaque 
patient atteint de PR. Les interventions thérapeutiques non médicamenteuses incluent notamment l'éducation thérapeutique ( $\mathrm{Cf}$ principe général $\mathrm{A})$, la gestion des comorbidités (Cf recommandation 15), les mesures psychologiques, sociales et professionnelles, la prise en charge non médicamenteuse de la douleur chronique et la prise en charge rééducative et chirurgicale [14]. Bien que les données de la littérature soient limitées, les experts sont d'avis que les traitements physiques, tels que la kinésithérapie, l'ergothérapie et la pédicuriepodologie apportent un bénéfice, notamment fonctionnel, aux patients atteints de PR [3,5]. L'utilisation d'orthèses adaptées et d'aides techniques peut représenter un intérêt pour certains patients. Les données concernant l'évaluation de l'efficacité d'une prise en charge psychologique sont rares mais le groupe d'experts considère que la proposition d'un soutien psychologique dans certains cas doit être envisagée. Il en est de même pour la prise en charge sociale et professionnelle. Enfin, il est recommandé d'informer tout patient atteint de PR de l'existence d'associations de malades, notamment dès que le diagnostic est posé, et de lui en communiquer les coordonnées, s'il le souhaite. En fonction des attentes de chaque patient, cette prise en charge globale peut se faire progressivement et pas nécessairement dès l'annonce du diagnostic.

Principe D: Le coût de la $\mathrm{PR}$, de ses conséquences et de ses traitements, à l'échelon individuel et sociétal, devrait être pris en considération dans les orientations thérapeutiques.

Ce principe a été très légèrement modifié par rapport à 2014, avec le retrait du mot « important » précédemment employé pour caractériser le coût de la PR. La notion de prise en compte du coût de la PR fait aussi partie des principes généraux des recommandations récentes de l'EULAR [2]. La décision thérapeutique doit se baser d'abord sur les données 
d'efficacité et de tolérance tout en prenant en compte le coût global de la prise en charge lié aux traitements. Les thérapeutiques ciblées ont accru les coûts directs de prise en charge de la PR. Cependant la maladie en elle-même est coûteuse du fait des coûts directs (hospitalisations, interventions chirurgicales) ou indirects (baisse de productivité, arrêts maladie, mises en invalidité) [15]. Du fait de leur efficacité, les thérapeutiques ciblées peuvent mener à une diminution de ces coûts directs ou indirects [16-18]. De plus, les stratégies actuelles de décroissance des thérapeutiques ciblées et l'arrivée des biosimilaires permettent une diminution individuelle des coûts de prescription des DMARDs (Disease Modifying Anti-Rheumatic Drugs) [19,20]. Dans ses dernières recommandations, l'EULAR rappelle qu'à tolérance et efficacité comparables, il faut choisir la stratégie thérapeutique la moins coûteuse [2].

\subsection{Recommandations}

\subsubsection{Diagnostic}

Recommandation 1: Le diagnostic de PR doit être: évoqué devant certains signes cliniques tels qu'un gonflement articulaire (arthrite clinique), une raideur matinale de plus de 30 min, une douleur à la pression transverse des mains ou des avants-pieds ; confirmé le plus tôt possible (idéalement dans les 6 semaines) par l'examen clinique du rhumatologue et par des examens biologiques (vitesse de sédimentation, C-réactive protéine, anticorps anti-protéines citrullinées (ACPA), facteurs rhumatoïdes) et d’imagerie (radiographies +/- échographie), après avoir éliminé les diagnostics différentiels.

La première partie de cette recommandation n'a pas été modifiée par rapport à 2014. L'existence de gonflements articulaires (synovites), surtout lorsqu'il y en a plusieurs et notamment au niveau des mains est évocatrice de PR, en particulier lorsqu'il s'y associe une 
raideur matinale de plus de 30 minutes et un «squeeze-test» positif (douleur à la pression transverse des métacarpophalangiennes (MCP) et/ou des métatarsophalangiennes (MTP)). Dans une publication récente, l'EULAR a défini certains paramètres devant faire suspecter un rhumatisme inflammatoire chez les patients présentant des arthralgies : durée d'évolution des symptômes inférieure à 1 an, atteinte des MCP, raideur matinale supérieure à 1 heure, prédominance matinale des symptômes, antécédent de PR chez un parent au premier degré, difficulté à fermer le poing, « squeeze-test » positif des MCP) [21].

La formulation de la deuxième partie de cette recommandation a légèrement été modifiée par rapport à 2014 afin de mentionner l'importance de l'examen clinique dans le diagnostic de PR et de faire apparaître la notion de délai optimal entre le début des symptômes et le diagnostic de PR. En effet, les données de la littérature indiquent que les patients vus rapidement par le rhumatologue après le début des symptômes ont un meilleur pronostic structural et un recours moins fréquent à la chirurgie orthopédique que ceux vus tardivement [4]. En se basant sur ces données et sur leur expérience, les experts du groupe de travail recommandent un délai optimal inférieur à 6 semaines entre le début des symptômes et la confirmation du diagnostic de PR. Par rapport aux recommandations de 2014, les experts souhaitaient mettre en avant le fait que l'examen clinique reste au premier plan pour détecter une PR. Ceci nécessite d'une part la formation des médecins généralistes aux signes évocateurs de rhumatisme inflammatoire débutant et une disponibilité du rhumatologue permettant un accès rapide [22]. L'échographie étant une technique d'imagerie non invasive, peu coûteuse et d'accès facile, elle reste utile pour aider au diagnostic de certains patients, pour la détection de synovites dans certains cas difficiles lorsqu'il y a un doute clinique (sujets obèses, atteinte des MTP, ...) ou pour quantifier le nombre d'articulations atteintes [23,24]. Cependant, au vu des données de la littérature, il a été considéré que l'échographie, bien qu'ayant une bonne sensibilité pour détecter les arthrites et les patients à risque de développer une PR, n'était pas systématique car 
pas assez spécifique du diagnostic de PR [3,4,25,26]. De même, les performances diagnostiques (du fait cette fois-ci d'une bonne spécificité mais d'une mauvaise sensibilité) de la recherche d'érosions échographiques chez les sujets présentant des arthrites ne permettent pas non plus de recommander l'utilisation systématique de l'échographie pour diagnostiquer une PR [25]. La pertinence clinique de l'IRM en tant que test diagnostic de PR semble limitée au vu des données actuelles, et de son coût $[3,4]$.

Sur le plan de l'imagerie, les radiographies standard des mains de face et des avants-pieds de face et de trois quarts gardent en revanche dans ces recommandations leur place en tant qu'examen de première intention pour aider au diagnostic de PR lorsqu'il existe des érosions typiques de PR. Le concept d'érosion typique de PR a été défini par l'EULAR : au moins trois articulations érosives parmi les MCP, les interphalangiennes proximales, les poignets et les MTP [27].

En l'absence d'érosion typique, la confirmation du diagnostic de PR repose donc sur un faisceau d'arguments cliniques et biologiques qui permettent aussi d'éliminer un éventuel diagnostic différentiel. Les examens biologiques à pratiquer devant une polyarthrite débutante ne sont pas tous consensuels. Les experts proposent de conserver les examens biologiques recommandés par la HAS en 2007, par la SFR en 2014 et par l'EULAR en 2016 et de réaliser au minimum un dosage de la protéine C-réactive, une mesure de la vitesse de sédimentation si possible, un hémogramme, un dosage des transaminases, une évaluation de la fonction rénale, une bandelette urinaire, un dosage du facteur rhumatoïde (FR), des anticorps anti-peptides cycliques citrullinés (ACPA) et des anticorps anti-nucléaires, un dépistage des hépatites B et C $[1,3,14]$. Les auto-anticorps ont un poids important pour le diagnostic. Dans la littérature, on retrouve ainsi une bonne performance des ACPA et du FR (quoique moindre) pour le diagnostic de PR [4]. De nombreuses méthodes de dosage des auto-anticorps existent mais les plus utilisées actuellement, et celles recommandées en France par la HAS en 2007, sont : pour 
le FR, le dosage de l'isotype IgM par technique ELISA (enzyme-linked immunosorbent assay) ou par néphélométrie ; pour les ACPA, le dosage des anticorps anti-CCP par technique ELISA [14].

Actuellement, les critères les plus utiles dans le contexte d'arthrite débutante restent les critères américano-européens (ACR/EULAR) conçus en 2010 comme critères de classification mais pouvant servir de référence en pratique en cas de diagnostic de PR difficile [28]. L'application de ces critères ne peut s'envisager qu'en présence d'au moins une synovite clinique et en l'absence d'autre diagnostic pouvant expliquer les symptômes. De plus, si un patient présente des lésions érosives typiques sur les radiographies standard, il peut d'emblée être classé comme ayant une PR, sans avoir à appliquer les critères ACR/EULAR 2010. Ces critères ont été élaborés en tant que critères de classification et pas en tant que critères de diagnostic mais ils peuvent être une aide intéressante pour le diagnostic précoce de PR à condition de ne pas remplacer le jugement du clinicien.

3.2.2. Objectif du traitement et organisation de la prise en charge

Recommandation 2: Dès que le diagnostic de PR est posé, un traitement de fond doit être débuté.

Cette recommandation n'a pas été modifiée par rapport à 2014.

L'instauration précoce, dès que possible, d'un traitement de fond adapté est capitale dans la prise en charge de la PR débutante qui est une «urgence thérapeutique ». Dans les recommandations 2016 de l'EULAR, les trois premiers mois d'évolution de la maladie constituent une «fenêtre d'opportunité thérapeutique » au cours de laquelle le traitement de fond devrait idéalement être introduit [3]. Les données actuelles de la littérature sont en effet 
concordantes et indiquent un meilleur pronostic clinique (obtention de la rémission, réponse au traitement, retentissement fonctionnel) mais aussi radiographique (limitation de la progression structurale, prévention de l'apparition des érosions) lorsque le traitement est débuté dans les trois mois suivant l'apparition des symptômes [5,29]. Par conséquent, l'instauration précoce d'un traitement de fond est aussi recommandée dès que possible et avant la confirmation du diagnostic en cas d'arthrite débutante indifférenciée à risque de progression vers une PR (niveau d'activité élevé, positivité des ACPA et/ou du FR, atteinte structurale) [3].

Recommandation 3. L'objectif du traitement est d'atteindre et de maintenir la rémission clinique ou au minimum la faible activité. La rémission clinique est définie par l'absence de signes et symptômes d'activité inflammatoire significative.

La première partie de cette recommandation a été modifiée en rajoutant la notion de «maintien de la rémission ». Elle a également été simplifiée par rapport à la recommandation de 2014 : « pour chaque patient, afin de prévenir la progression structurale et le handicap » a été retiré car ceci semblait implicite aux experts.

L'objectif du traitement de la PR est d'offrir aux patients la meilleure qualité de vie possible sur le long terme, en contrôlant leurs symptômes, en prévenant les lésions structurales et en leur permettant de mener une vie sociale et professionnelle normale. Il a été clairement montré que l'obtention de la rémission clinique et, à un moindre degré, du faible niveau d'activité est associée à une progression structurale et à un handicap fonctionnel moindres [30]. Certaines données suggèrent même que l'obtention d'une rémission précoce et prolongée diminuerait la mortalité des patients [31].

La rémission clinique est définie par l'absence de signes et symptômes d'activité inflammatoire significative. Ceci inclut les données de l'interrogatoire, de l'examen clinique 
et la biologie (syndrome inflammatoire) (voir aussi la recommandation 4). Les données actuelles de la littérature confirment que l'obtention de la rémission clinique est au moins aussi pertinente que l'obtention de la rémission échographique. Les études randomisées TASER et ARTIC et des données observationnelles en vraie vie (RABIODAM) n'ont pas montré de supériorité clinique ou structurale du suivi échographique par rapport au suivi clinique alors que l'utilisation systématique de l'échographie augmente le risque de surtraitement et les coûts [32-34].

La rémission clinique doit donc être le principal objectif thérapeutique pour tous les patients atteints de PR. Néanmoins, pour les patients ayant une PR ancienne avec d'importantes lésions structurales, la rémission est difficile à atteindre et le faible niveau d'activité est alors une alternative acceptable.

Dans tous les cas, le choix de l'objectif thérapeutique doit faire l'objet d'une décision partagée avec le patient. Un projet thérapeutique sera établi en prenant en compte les traitements antérieurement reçus, les comorbidités et les risques liés au patient et aux molécules. Ainsi, on pourra préférer un faible niveau d'activité à l'obtention d'une rémission à tout prix par exemple chez certains patients en échec de plusieurs lignes thérapeutiques ou à fort risque infectieux. A l'inverse, la présence de certaines comorbidités liées à l'inflammation chronique, telles que les pathologies cardiovasculaires, pourrait conduire à avoir des objectifs thérapeutiques plus stricts.

Le maintien de la rémission clinique est une notion importante puisqu'elle conditionne le suivi. Les recommandations EULAR 2016 considèrent la rémission comme persistante lorsqu'elle se maintient sur une période de 3 à 6 mois [30]. L'obtention d'une rémission persistante permet alors l'espacement du suivi des patients (voir recommandation 5). 
Recommandation 4. L'activité de la maladie doit être mesurée par des critères composites validés, incluant les indices articulaires.

Cette recommandation n'a pas été modifiée par rapport à 2014. Il existe différents critères composites validés permettant de mesurer l'activité de la maladie. L'utilisation de critères chiffrés permet de définir les objectifs thérapeutiques que sont la rémission et le faible niveau d'activité. Ces critères ne sont toutefois pas équivalents. Certains critères comme le Disease Activity Score 28 articulations ou DAS28 (rémission si DAS28-VS $<2,6$; faible niveau d'activité si 2,6 $\leq$ DAS28-VS $\leq 3,2$ ) sont dits permissifs puisque la rémission peut être atteinte malgré la persistance de plusieurs synovites cliniques [35]. Il est important de noter qu'aucun seuil de rémission n'a été défini pour le DAS28-CRP et que l'application des seuils établis pour le DAS28-VS conduit à une surestimation de la rémission clinique, le DAS28-CRP étant encore plus permissif que le DAS28-VS [36].

Les critères retenus par l'EULAR et l'ACR pour définir la rémission sont plus stricts. Ces critères sont :

- la rémission booléenne (nombre d'articulations douloureuses, nombre d'articulations gonflées, EVA activité et CRP (en mg/dl) tous $\leq 1)$;

- le Simplified Disease Activity Index ou SDAI (rémission si $\mathrm{SDAI} \leq 3,3$; faible niveau d'activité si $3,3<$ SDAI $\leq 11$ )

Le Clinical Disease Activity Index ou CDAI qui ne contient pas de critère biologique (rémission $\mathrm{CDAI} \leq 2,8$; faible niveau d'activité si $2,8<\mathrm{CDAI} \leq 10$ ) donne des résultats très comparables au SDAI $[35,36]$.

Contrairement à la rémission, pour le faible niveau d'activité, le DAS28-VS, le SDAI ou le CDAI semblent classer de façon similaire les patients [37].

Ces différents critères composites, comportant un indice articulaire, pourront être utilisés pour le suivi des patients atteints de PR. En plus de ces critères composites, l'évaluation de 
l'activité de la maladie devra aussi intégrer les différentes composantes du critère composite utilisé et la présence de synovites ou de ténosynovites au niveau des articulations non prises en compte par tous les critères, telles que les articulations des pieds.

Chez les patients ayant une PR récente traitée par DMARD synthétique conventionnel (csDMARD), les définitions EULAR/ACR de rémission (booléenne et SDAI) pourraient être préférentiellement utilisées, puisque la persistance de synovites chez les patients en rémission DAS28 est dans ce cas associée à une progression radiographique [38,39]. A l'inverse, les critères de rémission EULAR/ACR seront difficiles à atteindre chez les patients ayant une PR ancienne destructrice ou en présence de comorbidités interférant avec l'évaluation (fibromyalgie, arthrose ou pathologie responsable d'un syndrome inflammatoire biologique, etc.).

Dans le cas particulier des patients traités par inhibiteurs de la voie de l'IL-6, l'évaluation de la réponse clinique sur le DAS28-VS ou le DAS28-CRP est discutable car ces scores donnent une place importante au syndrome inflammatoire biologique qui diminue de façon quasiconstante du fait de l'effet biologique de l'inhibition de l'IL-6 [40].

Recommandation 5. Le suivi de la maladie par le rhumatologue doit être fréquent (1 à 3 mois) tant que la maladie est active. S'il n'y a pas d'amélioration dans les 3 mois suivant le début du traitement ou si l'objectif thérapeutique n'a pas été atteint à 6 mois, le traitement doit être ajusté.

La seule modification de cette recommandation par rapport à celle de 2014 est la mention du rhumatologue pour le suivi de la maladie.

Après avoir défini l'objectif thérapeutique, il est nécessaire de mettre en place un suivi rapproché (contrôle serré, «tight control») pour pouvoir atteindre rapidement cet objectif et 
ajuster, si nécessaire, le traitement. Il a en effet été clairement démontré qu'un suivi rapproché basé sur un critère composite d'activité tel que le DAS28 permet d'obtenir plus rapidement et plus fréquemment la rémission clinique qu'un suivi «classique » [30,41,42]. Un suivi tous les 1 à 3 mois (plutôt mensuel en cas de fort niveau d'activité) est recommandé jusqu'à l'obtention d'une rémission (ou faible niveau d'activité) prolongée c'est-à-dire confirmée à 36 mois. Le suivi pourra ensuite être espacé à tous les 6 mois (Figure 2).

La présence d'une amélioration significative à 3 mois est le premier élément de la réponse thérapeutique et sera évaluée par la variation du critère composite utilisé.

En plus du suivi clinique des critères composites articulaires d'activité, l'évaluation du retentissement fonctionnel par le Health Assessment Questionnaire (HAQ) est recommandée de façon annuelle puisque l'objectif de la prise en charge est notamment de prévenir le handicap fonctionnel. Bien que l'amélioration d'autres mesures basées sur le vécu du patient (patient-reported outcomes ou PRO) soit devenue un élément incontournable dans les études cliniques, l'utilisation des PRO comme critère d'intensification thérapeutique reste à évaluer.

Les recommandations françaises de 2014 proposaient de vérifier l'absence de progression structurale par des radiographies des mains et des pieds tous les 6-12 mois la première année puis tous les ans pendant 3-5 ans puis de façon plus espacée et en cas de changement thérapeutique. Les stratégies d'adaptation thérapeutique basées sur un contrôle serré avec utilisation de critères de rémission stricts comme ceux recommandés par l'EULAR et l'utilisation de thérapeutiques ciblées (DMARDs biologiques [bDMARDs] et DMARDs synthétiques ciblés [tsDMARDs]) ont clairement réduit la progression structurale des patients atteints de PR $[43,44]$. Les radiographies ont donc une place essentielle au début de la maladie, en cas d'activité clinique résiduelle au cours du suivi et lors des changements thérapeutiques mais sont probablement moins importantes chez les patients ayant une rémission clinique sans synovite résiduelle sous thérapie ciblée. L'utilisation d'autres 
méthodes d'imagerie telles que l'échographie et l'IRM pour suivre la progression structurale reste à démontrer.

\subsubsection{Traitement de $1^{\mathrm{e}}$ ligne (Figure 3)}

Recommandation 6. Le méthotrexate est le traitement de fond de première ligne chez les patients ayant une PR active avec une posologie initiale d'au moins $10 \mathrm{mg} / \mathrm{semaine}$ et une posologie optimale atteinte au maximum en 4 à 8 semaines.

Par rapport aux recommandations 2014, la posologie initiale minimale a été précisée.

Le MTX est le traitement de fond de première ligne chez les patients ayant une PR active. La posologie d'initiation doit être d'au moins $10 \mathrm{mg} / \mathrm{semaine}$ mais pourra être d'emblée à posologie maximale si le rhumatologue le juge nécessaire, l'objectif étant d'atteindre une posologie optimale comprise entre 15 et $30 \mathrm{mg} / \mathrm{semaine}$, en 4 à 8 semaines [45]. Certaines données suggèrent néanmoins une meilleure tolérance du MTX en cas d'augmentation progressive de la posologie [46]. La posologie optimale dépendra de l'efficacité, de la tolérance et du profil du patient. A noter que chez les patients d'origine asiatique, les posologies utilisées devront être moindres (posologie maximale autorisée de $20 \mathrm{mg} / \mathrm{semaine}$ en Chine et de $16 \mathrm{mg} / \mathrm{semaine}$ au Japon).

L'utilisation de la voie sous-cutanée peut être envisagée en cas de réponse insuffisante ou d'intolérance. La voie sous-cutanée permet en effet d'obtenir des concentrations sériques médicamenteuses équivalentes à 2,5-5 mg/semaine de plus que la voie orale pour une même posologie prescrite [47]. Néanmoins, le MTX par voie sous-cutanée a un coût plus élevé, ce qui pourra également être pris en considération. La prescription de MTX devra être accompagnée d'une supplémentation en acide folique d'au moins $10 \mathrm{mg} / \mathrm{semaine,} \mathrm{au} \mathrm{vu} \mathrm{des}$ données de la littérature [48]. 
L'association du MTX avec d'autres csDMARD ne semble pas supérieure à celle du MTX monothérapie dans la PR débutante [49-51] mais entraîne une moins bonne tolérance. Elle n'est donc pas recommandée en première intention.

La supériorité clinique et radiographique de l'association MTX + bDMARD par rapport au MTX en monothérapie en première ligne chez les patients atteints de PR a été confirmée dans plusieurs études récentes [52-55]. Néanmoins, le MTX était utilisé sans corticothérapie dans ces études. A l'inverse, dans l'étude IDEA, l'association MTX + infliximab ne faisait pas mieux que le MTX associé à une corticothérapie intraveineuse [56]. De plus, traiter tous les patients d'emblée par thérapie ciblée reviendrait à sur-traiter tous ceux qui atteindraient les objectifs thérapeutiques avec le traitement par MTX et donc à augmenter inutilement les risques encourus et les coûts. Il n'est donc pas recommandé d'utiliser une thérapie ciblée en première intention mais on associera facilement le MTX à une corticothérapie (voir recommandation 8$)$.

Recommandation 7. Chez les patients naïfs de traitement de fond, en cas de contre-indication au méthotrexate ou d'intolérance précoce, le leflunomide ou la sulfasalazine sont une alternative thérapeutique.

Cette recommandation n'a pas été modifiée par rapport à 2014. Les contre-indications au MTX sont relativement rares. Il s'agit avant tout de la grossesse ou de l'absence de contraception efficace, de l'insuffisance rénale sévère ou de pathologies hépatiques significatives. Les contre-indications pulmonaires restent exceptionnelles. Le leflunomide (LEF) et la sulfasalazine (SSZ) ont fait preuve de leur efficacité clinique et structurale [7]. Le LEF est utilisé à la dose de $20 \mathrm{mg} / \mathrm{j}$, mais est aussi contre-indiqué en cas de grossesse. La SSZ sera prescrite à posologie croissante jusqu'à atteindre la dose de 2 à $3 \mathrm{~g} / \mathrm{j}$. La SSZ pourra être utilisée en cas de grossesse. L'hydroxychloroquine (HCQ) peut être utilisée en combinaison 
avec d'autres csDMARD dans la PR d'autant que l'HCQ semblerait avoir un effet bénéfique métabolique et cardiovasculaire chez les patients atteints de PR [57]. Cependant, son utilisation en monothérapie n'est pas recommandée dans la PR du fait de sa faible efficacité clinique et de l'absence d'efficacité structurale [7].

Recommandation 8: Dans l'attente de l'efficacité d'un traitement de fond synthétique conventionnel, une corticothérapie orale ou injectable peut être proposée en respectant une dose cumulée faible, si possible sur une période maximale de 6 mois. La corticothérapie sera diminuée et arrêtée aussi rapidement que possible.

Par rapport à 2014, il a été précisé que les corticoïdes peuvent être proposés lors de l'instauration des traitements de fond synthétiques conventionnels (csDMARDs), par opposition aux traitements de fond ciblés (biologiques ou synthétiques) avec lesquels l'association initiale à une corticothérapie est considérée comme plus optionnelle. De la même façon qu'en 2014, la nécessité de respecter une durée de corticoïdes courte, inférieure à 6 mois, afin d'aboutir à une dose cumulée faible a été rappelée. Les différentes voies d'administration possible de la corticothérapie (orale ou injectable) ont été précisées dans la recommandation. Enfin, la formulation actuelle de cette recommandation insiste sur le fait que la corticothérapie doit non seulement être diminuée mais aussi arrêtée le plus rapidement possible.

Les données actuelles de la littérature confirment que les corticoïdes ont une efficacité à la fois clinique et structurale et gardent donc une place dans la prise en charge de la PR $[5,7,58,59]$. De plus, les connaissances actuelles sont en faveur d'une tolérance globalement bonne des corticoïdes lorsqu'ils sont utilisés à court terme et à de faibles posologies $[5,8,58,60,61]$. Cependant, malgré le biais d'indication chez les patients ayant une forte activité de la maladie, les nouvelles études continuent aussi à apporter des preuves de la 
toxicité des corticoïdes au long cours, même à faible posologie, notamment sur les risques cardiovasculaires, infectieux et ostéoporotiques $[5,8,62,63]$. En se basant sur l'évaluation de ce rapport bénéfice-risque des corticoïdes, le groupe d'experts considère donc que l'utilisation des corticoïdes ne peut s'envisager qu'à la plus petite dose cumulée possible et pour la plus courte durée possible. La notion de dose cumulée sous-entend qu'initialement de fortes doses de corticoïdes peuvent être employées, que ce soit par voie orale, intra-musculaire ou intraveineuse, afin de diminuer ensuite plus rapidement la corticothérapie [58,64,65]. Les injections intra-articulaires de corticoïdes semblent aussi être bénéfiques, en association aux csDMARDs [5]. Le groupe de travail considère que la durée maximale souhaitable de corticothérapie doit être de 6 mois. Certains experts du groupe de lecture ont proposé que cette durée maximale soit réduite à 3 mois. En accord avec les dernières recommandations de l'EULAR, le groupe d'experts de la SFR recommande que la corticothérapie soit considérée lors de toute initiation de csDMARD, que ce soit en première ligne ou en cas de changement de traitement, bien que l'effet bénéfique des corticoïdes sur le contrôle de la maladie soit essentiellement étayé dans les PR débutantes [2]. Enfin, le groupe d'experts recommande que dans tous les cas la prescription de corticoïdes soit basée sur une évaluation de la balance bénéfice-risque chez chaque patient et s'accompagne systématiquement d'une prévention des effets secondaires (Cf recommandation 15).

\subsubsection{Traitement de $2^{\text {nde }}$ ligne et plus (Figure 3 )} traitement doit être optimisé. 
En présence de facteurs de mauvais pronostic, l'addition d'une thérapeutique ciblée (biologique ou synthétique) peut être proposée : anti-TNF, abatacept, inhibiteurs de la voie de l'IL6, inhibiteurs de JAK et dans certaines circonstances rituximab.

En l'absence de facteurs de mauvais pronostic, une rotation pour un autre traitement de fond synthétique conventionnel (LEF, SSZ) ou bien une association de traitements de fond synthétiques conventionnels peuvent être proposées ; en cas d'échec (ou de contre-indication), une thérapeutique ciblée (biologique ou synthétique) doit être envisagée.

Par rapport à 2014, la recommandation a été reformulée pour mettre en avant la nécessité d'optimiser le traitement de fond en cas de réponse insuffisante («doit être optimisé »a remplacé «peut être proposé »). Par ailleurs, la référence aux biomédicaments a été élargie aux thérapeutiques ciblées qui font référence aux bDMARDs et aux tsDMARDs. Enfin, le détail de la combinaison de csDMARD (MTX/SSZ/HCQ) a été retiré.

En l'absence d'amélioration suffisante à 3 mois ou si l'objectif thérapeutique n'a pas été atteint à 6 mois, le traitement doit être optimisé (voir recommandations 3 et 5). La stratégie d'optimisation dépend de la présence ou non des facteurs de mauvais pronostic ou de mauvaise réponse suivants :

- présence d'érosions précoces

- présence de facteur rhumatoïde et d'ACPA, particulièrement en cas de taux élevés $(\geq 3 \mathrm{~N})$

- persistance d'une activité de la maladie modérée à forte malgré un traitement par csDMARD avec niveau élevé de VS et CRP et/ou nombre élevé d'articulations gonflées

- $\quad$ échec de $\geq 2$ csDMARDs

Les facteurs de mauvais pronostic listés ci-dessus sont les facteurs identifiés par les données récentes de la littérature comme étant des facteurs de risque de progression structurale [66,67]. Il n'existe pas d'étude spécifiquement conçue pour comparer l'efficacité des csDMARD à celle des thérapeutiques ciblées en fonction de la présence ou non de facteurs de 
mauvais pronostic. Néanmoins, la présence d'érosions précoces, d'autoanticorps et d'un niveau élevé d'activité a été très largement associée à une progression structurale plus importante [66] et les thérapeutiques ciblées ont un effet structural supérieur aux csDMARDs. Des analyses post-hoc confirment que les patients ayant des facteurs de mauvais pronostic bénéficient plus de l'adjonction d'un bDMARD au MTX que les patients sans $[67,68]$. L'utilisation d'un autre csDMARD en cas d'activité modérée à forte malgré un traitement par csDMARD a en effet montré ses limites en pratique courante [69]. Par rapport aux recommandations précédentes, l'échec à $\geq 2$ csDMARDs a été rajouté. Il ne s'agit pas en soi d'un facteur de mauvais pronostic mais plutôt d'un facteur de mauvaise réponse. Les experts considèrent en effet qu'en cas d'échec à 2 csDMARDs, la probabilité de réponse à un $3^{\mathrm{e}}$ csDMARD est très faible et qu'il s'agit donc d'une indication à une thérapeutique ciblée [68].

En présence de facteurs de mauvais pronostic, toutes les thérapeutiques ciblées peuvent être utilisées (bDMARD ou tsDMARD). En effet, les études face/face [70-72] et les métaanalyses n'ont pas montré de différence consistante d'efficacité entre les différentes thérapeutiques ciblées utilisées en combinaison avec le MTX [6,73,74]. Parmi les thérapeutiques ciblées, les bDMARDs pourront être préférés du fait du plus long recul d'utilisation et des données de tolérance à long terme issues des registres [8,75]. Néanmoins, les données de tolérance des tsDMARD sont rassurantes [76,77] et le baricitinib pourrait être plus efficace que l'adalimumab en association au MTX [72]. Le rituximab n'a pas l'AMM en première ligne de thérapie ciblée mais pourra être utilisé dans les cas particuliers que sont : les antécédents personnels de cancer de moins de 5 ans, la tuberculose latente avec impossibilité d'antibioprophylaxie et les antécédents personnels de lymphome et de pathologies démyélinisantes, le rituximab ayant montré son efficacité dans ces situations [78,79]. L'abatacept peut aussi être une alternative en cas de pathologie démyélinisante [80]. 
En l'absence de facteur de mauvais pronostic, le remplacement ou l'ajout d'autres csDMARDs sera proposé. Dans ce cas, comme pour la $1^{\mathrm{e}}$ ligne de traitement, l'association à une corticothérapie pourra aider au contrôle rapide de l'activité. Bien que les combinaisons de csDMARD (par exemple MTX/SSZ/HCQ ou MTX/SSZ ou MTX/HCQ) n'aient pas montré de supériorité claire par rapport au MTX monothérapie, lorsque les deux groupes sont en association à une corticothérapie (voir recommandation 6), elles pourraient avoir une place chez les patients en cas d'échec au MTX en l'absence de facteur de mauvais pronostic ou en cas de contre-indication, notamment un problème infectieux, à une thérapie ciblée $[81,82]$.

En cas d'arrêt pour intolérance au MTX avant 3 mois de traitement, on préférera un changement de csDMARD (LEF, SSZ) à l'instauration d'une thérapie ciblée, comme le stipule la recommandation 7 .

Recommandation 10. Toutes les thérapeutiques ciblées (biologiques ou synthétiques) doivent être utilisées préférentiellement en association avec le MTX.

Par rapport aux recommandations 2014, le terme «thérapeutiques ciblées » a remplacé celui de «biomédicaments » pour inclure les tsDMARDs.

La combinaison d'une thérapeutique ciblée avec le MTX est cliniquement et radiographiquement plus efficace que la thérapeutique ciblée en monothérapie pour tous les bDMARDs [6,83], y compris pour le tocilizumab avec des résultats numériquement meilleurs pour la combinaison [84-86]. C'est également le cas des inhibiteurs de JAK. Ainsi, dans ORAL strategy, l'association tofacitinib + MTX induit une réponse ACR50 à 6 mois chez $46 \%$ des PR contre $38 \%$ pour le tofacitinib monothérapie [70]. Dans RA-BEGIN, l'association baricitinib + MTX induit une rémission DAS28-VS à 12 mois chez $30 \%$ des patients contre $22 \%$ pour le baricitinib monothérapie, avec une efficacité structurale également numériquement supérieure pour la combinaison $(79,9 \%$ vs $68,8 \%$ de non- 
progresseurs radiographiques à 1 an) [87]. Tous les bDMARDs et les tsDMARDs doivent donc être utilisés en association avec le MTX quand cela est possible.

L'étude CONCERTO a montré qu'associer à l'adalimumab $10 \mathrm{mg} / \mathrm{semaine}$ de MTX était aussi efficace qu'associer $20 \mathrm{mg} / \mathrm{semaine}$ de MTX et plus efficace qu'associer 2,5 et 5 mg/semaine de MTX [88]. Dans l'étude MUSICA, l'association adalimumab + MTX 20 $\mathrm{mg} / \mathrm{semaine}$ était plus efficace que l'association adalimumab + MTX 7,5 mg/semaine [89]. Ainsi, chez les patients recevant une thérapeutique ciblée, la diminution de posologie du MTX pourra se discuter en maintenant une posologie d'au moins $10 \mathrm{mg} / \mathrm{semaine}$.

En cas de contre-indication ou intolérance au MTX, on pourra associer à la thérapeutique ciblée du LEF ou de la SSZ. Plusieurs études ont montré une efficacité comparable des associations anti-TNF + MTX et anti-TNF + LEF [90,91]. D'après le registre anglais British Society of Rheumatology Biologics Register for Rheumatoid Arthritis (BSRBR), la combinaison avec de la SSZ est associée à un risque moindre d'arrêt de l'anti-TNF que la monothérapie, bien que ce risque reste plus important qu'avec le MTX [92].

En cas d'impossibilité d'utiliser un csDMARD en association, il faudra éviter d'initier un anticorps monoclonal anti-TNF en monothérapie. En effet, il a été montré de manière constante que les anticorps monoclonaux anti-TNF utilisés en monothérapie avaient une moindre efficacité clinique que la combinaison au MTX, en partie liée à l'immunisation anti médicament, plus fréquente en l'absence de MTX [93]. Les données les plus solides concernant l'efficacité des thérapeutiques ciblées en monothérapie concernent les inhibiteurs du récepteur de l'IL-6 et les tsDMARD. Le tocilizumab et les inhibiteurs de JAK en monothérapie donnent des taux de répondeurs proches de ceux obtenus avec la combinaison au MTX dans les études randomisées comme dans les cohortes [70,87,94,95]. Par ailleurs les études ADACTA et MONARCH ont montré une supériorité du tocilizumab et du sarilumab par rapport à l'adalimumab en monothérapie [96,97]. Dans le registre TOCERRA, l'efficacité 
et le maintien thérapeutiques du tocilizumab monothérapie sont similaire à ceux des anti-TNF associés au MTX [95,97].

L'etanercept et l'abatacept ne posent pas de problème d'immunogénicité. Dans certains essais randomisés contrôlés, l'etanercept en monothérapie a montré une efficacité clinique mais significativement moindre que la combinaison avec le MTX et une supériorité sur le plan structural par rapport aux csDMARDs [98,99]. Certaines données observationnelles suggèrent une efficacité de l'abatacept mais celui-ci n'a pas l'autorisation de mise sur le marché en monothérapie [100,101].

Ainsi, chez les patients qui ne peuvent pas recevoir de csDMARD, les inhibiteurs du récepteur 1'IL-6 et les inhibiteurs de JAK semblent présenter un avantage par rapport aux autres thérapeutiques ciblées et surtout par rapport aux anticorps monoclonaux anti-TNF dont l'initiation en en monothérapie doit être évitée.

Recommandation 11. Les patients en échec d'une première thérapeutique ciblée (biologique ou synthétique) doivent être traités par une autre thérapeutique ciblée. En cas d'échec primaire, un changement de mode d'action peut être privilégié.

Par rapport aux recommandations de 2014, le terme «thérapeutiques ciblées » a remplacé celui de «biomédicaments » pour inclure les tsDMARDs. La notion d'intérêt à changer de mode d'action en cas d'échec primaire a été rajoutée.

Après échec à un $1^{\mathrm{e}}$ anti-TNF, la plupart des études retrouvent une efficacité comparable des différentes thérapeutiques ciblées, que ce soit un $2^{\mathrm{e}}$ anti-TNF ou d'un autre mode d'action [6,102,103]. L'étude française ROC montrait une réponse EULAR supérieure lorsqu'un autre mécanisme d'action était utilisé après échec d'un anti-TNF [104]. Néanmoins cette différence était essentiellement expliquée par une forte réponse EULAR chez les patients traités par tocilizumab (48\% des patients du groupe « autre mode d'action »), qui diminue constamment 
les paramètres inflammatoires biologiques. On pourra donc utiliser indifféremment un médicament ayant le même mode d'action (ex. un $2^{\mathrm{e}}$ anti-TNF) ou un mode d'action différent. A noter néanmoins qu'il n'y a pas actuellement dans la littérature de données permettant d'évaluer l'efficacité d'un $2^{\mathrm{e}}$ inhibiteur de JAK après échec d'un premier tsDMARD, ni de données sur l'efficacité d'un $2^{\mathrm{e}}$ inhibiteur de l'IL-6 après échec du tocilizumab.

Dans le cas particulier de l'échec primaire, c'est-à-dire en l'absence d'amélioration suffisante à 3 mois ou d'obtention de la cible thérapeutique à 6 mois, un changement de mode d'action semble devoir être privilégié. Les données de cohorte suggèrent en effet la supériorité d'une thérapeutique ciblant un autre mode d'action en cas d'échec primaire à un $1^{\mathrm{e}}$ anti-TNF [105]. Dans l'étude EXXELERATE (certolizumab + MTX versus adalimumab + MTX), l'utilisation $\mathrm{du} 2^{\mathrm{e}}$ anti-TNF chez les patients en échec primaire au $1^{\mathrm{e}}$ anti-TNF donne cependant des taux de répondeurs satisfaisants bien que moins bons qu'avec le $1^{\mathrm{e}}$ anti-TNF [106].

Il est important de rappeler l'importance d'évaluer l'observance thérapeutique des patients, la mauvaise observance étant une cause fréquente d'échec thérapeutique. On sait par exemple que l'adhérence au MTX est très variable [107]. Elle est influencée par les croyances des patients qui peuvent elles-mêmes être influencées par l'éducation thérapeutique [108].

\subsubsection{Gestion de la rémission}

Recommandation 12 : En cas de rémission persistante et sans corticoïdes, une décroissance de la thérapeutique ciblée (biologique ou synthétique) doit être envisagée.

En 2014, cette recommandation était moins restrictive vis-à-vis de la corticothérapie en notifiant «arrêt des corticoïdes (ou décroissance $\leq 5 \mathrm{mg} / \mathrm{j}$ ) ». Le groupe d'expert a choisi d'indiquer uniquement « sans corticoïdes » dans la recommandation actuelle afin de signifier l'importance d'essayer d'obtenir le sevrage en corticoïdes en cas de rémission. De plus, dans 
cette recommandation, le terme «thérapeutique ciblée » remplace le terme «biomédicament » utilisé dans la recommandation 2014 afin de prendre en compte les tsDMARD. Enfin, le groupe d'expert a choisi d'être plus directif que précédemment en notifiant que la décroissance « doit » (au lieu de «peut » en 2014) être envisagée.

La rémission persistante est définie pour la plupart des auteurs, par le maintien de la rémission sur une période de 3 à 6 mois [1,2]. L'inflammation et la destruction articulaire pouvant être dissociées, certains patients continuent de progresser sur le plan structural malgré une rémission clinique, et il faut donc répéter les radiographies avant et pendant la décroissance thérapeutique afin de s'assurer de l'absence d'évolution des lésions radiographiques [109]. Lorsque cette rémission persistante a été obtenue, avant d'envisager une décroissance des traitements de fond, la corticothérapie devra au préalable être arrêtée. Dans certains cas particuliers, et notamment chez les patients atteints de PR anciennes, cette décroissance pourra s'envisager même si l'arrêt complet des corticoïdes n'a pas été possible, sous réserve que la corticothérapie ait été réduite à une dose minimale (inférieure ou égale à $5 \mathrm{mg} / \mathrm{j})$. L'utilisation de l'échographie pour vérifier l'absence d'activité résiduelle infraclinique n'a pas montré à ce jour sa pertinence pour guider la décroissance (voir recommandation 3).

Les études indiquent qu'en cas d'arrêt brutal d'un bDMARD, une faible proportion de patients maintient la rémission ou le faible niveau d'activité [6]. De ce fait, cette stratégie n'est pas recommandée. En revanche, les données actuelles de la littérature indiquent que la décroissance progressive des thérapeutiques ciblées (diminution des doses ou espacement des administrations), et adaptée selon l'activité de la maladie, permet à une proportion significative de patients de maintenir la rémission ou à défaut un faible niveau d'activité de la maladie [6]. Ainsi, les études d'extension à 3 ans des essais contrôlés randomisés STRASS et DRESS publiées récemment confirment que l'évolution des paramètres d'activité de la 
maladie (cliniques, et radiographiques pour STRASS) ne diffère pas entre les patients en rémission (STRASS) ou ayant un faible niveau d'activité de la maladie (DRESS) ayant réduit leur traitement anti-TNF et ceux l'ayant maintenu à la même posologie [110,111]. Les études concernant la décroissance des biomédicaments non anti-TNF et des inhibiteurs de JAK restent encore rares mais leurs conclusions semblent être comparables [112-114]. Dans les études, la reprise de la thérapeutique ciblée à sa posologie ou à sa fréquence d'administration initiale en cas de poussée, permet dans la majorité des cas (> 80\%) le retour à un contrôle adéquat de l'activité de la PR [112,114,115].

Il est à noter que la population de la plupart des études évaluant les stratégies de décroissance des bDMARDs et des tsDMARD était constituée de patients traités en combinaison avec un csDMARD. Par conséquent, la décroissance des thérapeutiques ciblées chez les patients en rémission doit s'envisager préférentiellement chez les patients recevant aussi un csDMARD. Parmi ces facteurs prédictifs, on peut aussi noter la rapidité d'atteinte de la rémission ainsi que la durée et la profondeur de cette rémission [116,117]. L'état de faible activité de la maladie ne semble en revanche pas être suffisant pour envisager de débuter une décroissance thérapeutique [116].

Recommandation 13 : En cas de rémission persistante prolongée sans thérapeutique ciblée et sans corticoïdes, une réduction progressive des traitements de fond conventionnels synthétiques peut être envisagée.

La formulation de cette recommandation a été légèrement modifiée par rapport à 2014 pour indiquer que la décroissance des traitements de fond conventionnels synthétiques doit s'envisager chez les patients sans corticoïdes et ne recevant pas (ou plus) de thérapeutique ciblée car sinon la décroissance de cette dernière doit être considérée en priorité. De plus, la 
partie de phrase «dans le cadre d'une décision médicale partagée entre le patient et le médecin » a été retirée car le groupe d'expert a considéré que cette notion a été rappelée dans le principe général A et doit donc s'appliquer pour l'ensemble des recommandations.

La décroissance des csDMARDs peut être proposée aux patients qui sont en rémission persistante prolongée sans corticoïdes (Cf recommandation 12). Peu d'études ont évalué de façon spécifique la décroissance des csDMARDs [116]. De façon similaire à ce qui a été observé avec les thérapeutiques ciblées, la décroissance progressive adaptée à l'activité de la maladie semble préférable à un arrêt brutal qui expose à un risque élevé de rechutes [118,119]. La rémission sans traitement étant rarement obtenue, l'arrêt brutal des csDMARDs ne peut s'envisager que dans des cas particuliers [116].

\subsubsection{Prise en charge globale}

Recommandation 14: Le choix et l'adaptation thérapeutiques doivent intégrer d'autres facteurs que la mesure de l'activité de la maladie comme la progression structurale, les maladies associées, la tolérance des traitements, l'adhésion et l'avis du patient.

Par rapport à 2014, le mot « adhésion » a été rajouté à cette recommandation.

Il est en effet capital que le patient adhère au projet thérapeutique afin d'assurer non seulement une observance optimale mais aussi une implication et une persistance dans sa prise en charge médicamenteuse et non médicamenteuse. Des recommandations concernant l'adhésion médicamenteuse des patients atteints de rhumatismes inflammatoires chroniques ont ainsi été établies en 2017 par un groupe de travail français lors des Rencontres d'Experts en Rhumatologie (Puyraimond-Zemmour D et al., abstract SAT0691, EULAR 2018). L'évaluation de l'adhésion à chaque consultation y est notamment recommandée et, en cas de détection de non-adhésion, une intervention spécifique (éducation thérapeutique, entretien 
motivationnel) doit être mise en place. L'évaluation de la tolérance des traitements prescrits doit aussi être intégrée dans le choix et l'adaptation des thérapeutiques prescrites car il s'agit d'un élément clé dans l'adhésion médicamenteuse.

Cette recommandation rappelle de plus que toutes les caractéristiques du patient, telles que les comorbidités ou un désir de grossesse, doivent être prises en compte dans les décisions thérapeutiques. Ainsi, les comorbidités peuvent avoir un impact sur le choix de l'objectif thérapeutique (voir recommandation 3) et la réponse thérapeutique chez les patients atteints de PR, que ce soit pour des raisons d'interactions physiopathologiques entre les différentes maladies associées, d'influence sur l'évaluation de l'activité de la PR par le patient ou du fait de la polymédication [120].

Enfin, comme vu dans la recommandation 12, certains patients progressant sur le plan structural malgré une maladie cliniquement peu active, il est nécessaire de surveiller et de prendre en compte les lésions radiographiques lors des décisions thérapeutiques.

Recommandation 15. Un dépistage et une évaluation périodique des comorbidités, de leurs facteurs de risque et de leur prise en charge doivent être réalisés. La prise en charge doit être associée à des conseils d'hygiène de vie (activité physique régulière, arrêt du tabac, alimentation équilibrée, etc.) et à la mise à jour des vaccinations.

L'ancienne recommandation 15 , qui ne faisait que citer les comorbidités, a été remplacée par le principe général C. Cette recommandation a été rajoutée pour détailler la prise en charge de comorbidités.

Les patients atteints de PR ont un risque cardiovasculaire $(\mathrm{CV})$ élevé, comparable à celui des patients diabétiques [121]. Ce sur-risque est expliqué par l'inflammation chronique et l'utilisation de certains traitements comme les corticoïdes et les AINS [122]. Le contrôle de 
l'inflammation par les DMARDs et en particulier les thérapies ciblées, est associé à une réduction du risque $\mathrm{CV}$ [122].

La prise en charge du risque CV passe donc par : i) l'évaluation du risque CV et le dépistage des facteurs de risque CV traditionnels (tabagisme, diabète, hypertension artérielle, dyslipidémie, obésité); ii) la prise en charge de ces facteurs de risque CV traditionnels iii) le contrôle de l'inflammation. Comme proposé par l'EULAR, il apparaît souhaitable que le rhumatologue soit responsable de l'organisation du dépistage et de la prise en charge du risque $\mathrm{CV}$ des patients atteints de PR [123]. L'évaluation du risque CV devrait idéalement utiliser le score Heart-SCORE®. Le niveau de risque CV déterminera la cible thérapeutique du LDL-cholestérol, la nécessité ou non de référer le patient à un cardiologue et la fréquence du dépistage. L'EULAR suggère également l'utilisation de l'échographie doppler des troncs supra-aortiques pour classer les patients ayant des plaques carotidiennes comme étant à haut risque. Ces plaques sont retrouvées chez près de $60 \%$ des patients ayant une PR [124].

Le dépistage et la prise en charge des comorbidités ne se limitent pas à l'évaluation du risque $\mathrm{CV}$. Les patients atteints de PR ont également une augmentation des risques infectieux, pulmonaire, néoplasique, digestif, ostéoporotique et de dépression induits par la maladie et les traitements utilisés [125]. Par ailleurs, ils sont moins bien dépistés que la population générale. On vérifiera notamment la mise à jour des dépistages néoplasiques comme pour la population générale, l'état bucco-dentaire et les vaccinations. En plus de ceux de la population générale, les patients atteints de PR doivent être vaccinés contre la grippe annuellement, la diphtérietétanos-poliomyélite tous les 10 ans et le pneumocoque tous les 5 ans [126]. Les patients non antérieurement vaccinées reçoivent la primo-vaccination pneumococcique par une dose de vaccin conjugué 13-valent suivie au moins 8 semaines plus tard d'une dose de vaccin non conjugué 23-valent; les patients qui n’ont reçu antérieurement que le vaccin non conjugué 23valent pourront recevoir une injection du vaccin conjugué 13-valent si la vaccination 
antérieure remonte à plus de 1 an ou sinon une injection ultérieure du vaccin non conjugué 23-valent au minimum 5 ans après la date de l'injection du vaccin non conjugué 23-valent; les personnes déjà vaccinées suivant la séquence vaccin conjugué 13-valent - vaccin non conjugué 23-valent pourront recevoir une nouvelle injection du VPP23 en respectant un délai de 5 ans après la précédente injection de ce même vaccin. Le reste du calendrier vaccinal est identique à celui de la population générale.

Un bon état bucco-dentaire diminue le risque infectieux et, du fait des liens entre parodontopathies et PR [127,128], pourrait avoir un effet bénéfique pour la maladie [129].

Différentes modalités de dépistage peuvent être proposées. Soit le rhumatologue peut l'organiser au cours de ses consultations, en répartissant le dépistage sur plusieurs consultations ; soit le dépistage peut être structuré au sein des hôpitaux ou des cliniques par exemple, sur une hospitalisation de jour [130]; soit le dépistage peut être en partie fait par une infirmière spécialisée [131]. Les dernières recommandations applicables en France et en Europe en matière de dépistage $\mathrm{CV}$ et des autres comorbidités ont été regroupés dans un document pour faciliter leur application par les rhumatologues [132].

La prise en charge doit être associée à des conseils d'hygiène de vie. Les programmes visant à améliorer l'hygiène de vie ont en effet montré un effet bénéfique sur le risque CV [133]. On recommandera une activité physique régulière qui pourrait également avoir un impact positif sur les douleurs des patients, leur qualité de vie et leur moral [134]. L'arrêt du tabac sera conseillé pour diminuer le risque CV [135]. L'arrêt du tabac pourrait également être associé à une moindre activité de la maladie et à une meilleure réponse thérapeutique [136,137]. Enfin, une alimentation équilibrée est recommandée. Le régime méditerranéen et la consommation d'oméga-3 (poissons gras, d'huile de poisson ou suppléments) sont associés à une diminution du risque CV [138] et à un petit effet bénéfique sur l'activité de la PR [139,140]. Ils seront donc encouragés. A l'inverse, les régimes d'exclusion tels que le régime sans gluten et le 
régime sans lactose n'ont pas fait la preuve de leur efficacité clinique (ni même pré-clinique sur des modèles animaux) mais pourraient être associés à un sur-risque $\mathrm{CV}$ pour le régime sans gluten $[141,142]$ et à un sur-risque d'ostéoporose pour le régime sans lactose. Ils doivent donc être évités. Ainsi, les patients atteints de PR doivent être pris en charge dans leur globalité (Figure 4). 


\section{Discussion}

La recherche apporte en permanence de nouvelles données dans le domaine de la PR, que ce soit pour le développement de nouveaux traitements, l'élaboration de stratégies thérapeutiques ou la prise en charge non médicamenteuse. Du fait de cette dynamique, il semble nécessaire de mettre à jour régulièrement les recommandations de prise en charge afin d'apporter aux professionnels de santé impliqués dans la gestion de cette pathologie des recommandations adaptées aux connaissances actuelles. C'est pourquoi la SFR a décidé d'actualiser en 2018 les recommandations qui avaient été établies en 2014.

Ce nouvel ensemble des recommandations de la SFR pour la prise en charge de la PR comporte 4 principes généraux et 15 recommandations couvrant la globalité de la gestion de la PR, de la phase diagnostique à la phase thérapeutique, médicamenteuse et nonmédicamenteuse ainsi qu'au suivi des patients et à la prise en charge des comorbidités.

De nombreux points sont similaires à ceux abordés en 2014, notamment en ce qui concerne les principes généraux, à savoir la place centrale du rhumatologue dans la prise en charge de la PR, la nécessité d'une alliance thérapeutique entre le patient et son rhumatologue, le besoin d'une prise en charge globale de la PR et des maladies associées (incluant non seulement les traitements médicamenteux mais aussi les mesures non-médicamenteuses telles que la rééducation et l'éducation thérapeutique) et la prise en compte du coût à la fois individuel et sociétal de la PR. Enfin, de la même façon qu'en 2014, l'importance du diagnostic et du traitement les plus précoces possibles de la PR est rappelée.

Les recommandations 2018 diffèrent aussi par certains aspects des recommandations 2014. Ainsi, les nouvelles thérapeutiques ciblées synthétiques étant arrivées récemment sur le marché français, elles sont mentionnées dans les recommandations. Le groupe d'experts a décidé de placer ces nouvelles molécules anti-JAK au même rang que les biomédicaments. 
Par rapport à 2014, la notion de choix préférentiel d'un traitement ayant un mode d'action différent en cas d'échec primaire à une thérapeutique ciblée a été abordée. Enfin, la place de l'hygiène de vie et de la gestion des comorbidités en tant que domaine à part entière de la prise en charge de la PR a été renforcée.

La plupart des recommandations élaborées ont un niveau d'évidence et d'accord élevé, et une force de recommandation importante. A l'exception du principe général $\mathrm{B}$, le niveau d'accord avec chaque principe et recommandation était moins élevé pour les membres du groupe de lecture que pour les membres du groupe de travail (Tableau 2) et ceci s'explique sans doute en partie par l'évaluation des recommandations sans le détail du texte. En effet, les commentaires faits par le groupe de lecture portaient essentiellement sur des points qui avaient été discutés par les experts et qui ont été développés dans le corps du texte des recommandations. Les recherches liées à la $\mathrm{PR}$ se poursuivant, il est probable que ces recommandations devront de nouveau être actualisées d'ici quelques années.

\section{Déclaration de liens d'intérêts :}

C.Daien a reçu des honoraires de Abbvie, BMS, Novartis, Pfizer, Roche-Chugai, Sanofi, UCB

C.Hua a reçu des honoraires de Abbvie, BMS, Novartis, Pfizer

C.Gaujoux-Viala a reçu des honoraires de Abbvie, BMS, Celgene, Janssen, Lilly, Medac, MSD, Nordic Pharma, Novartis, Pfizer, Sanofi, Schering, Roche- Chugai, UCB

A.Cantagrel a reçu des honoraires de AbbVie, BMS, Merck, Nordic Pharma, Novar-tis, Pfizer, Roche-Chugai, UCB

M.Dougados a participé comme consultant à des réunions organisées par les laboratoires Pfizer, Abbvie, Roche, UCB, BMS, Lilly, Janssen, Novartis et son service a reçu des bourses 
de recherche des laboratoires Pfizer, Abbvie, Roche, UCB, BMS, Lilly, Janssen, Novartis.

B.Fautrel a reçu des honoraires de AbbVie, BMS, MSD, Nordic Pharma, Pfizer,RocheChugai, UCB

X.Mariette a reçu des honoraires de BMS, Janssen, Novartis, Pfizer, UCB

C.Richez a reçu des honoraires de Abbvie, BMS, Lilly, Medac, Merck, Nordic Pharma, Pfizer, Roche-Chugai, Sanofi, UCB

A.Saraux a reçu des honoraires de Abbvie, BMS, Lilly, Merck, Pfizer, Roche-Chugai, Sanofi, UCB

D.Wendling a reçu des honoraires de AbbVie, BMS, MSD, Pfizer, Roche Chugai, Amgen, Nordic, UCB, Sobi, Sanofi Aventis, Novartis, Janssen, Hospira, Celgene , Lilly, Sandoz

L.Gossec a reçu des honoraires de Abbvie, BMS, Celgene, Janssen, Lilly, Novartis, Pfizer, Sanofi, UCB.

B.Combe a reçu des honoraires de Abbvie, BMS, Janssen, Lilly, Merck, Pfizer, Roche-Chugai, Sanofi, UCB

M.Dubremetz, N.Nayral, G.Thibaud : Aucun conflit d'intérêt

\section{Remerciements :}

Nous remercions le groupe de lecture qui a relu et commenté ces recommandations :

Baudens G, Beauvais C, Berenbaum F, Berthelot J-M, Boumier P, Brocq O, Constantin A, Devauchelle-Pensec V, Dixneuf V, Fayet F, Flipo R-M , Gaudin P, Goeb V, Goupille P, Grange L, Hudry C, Jousse-Joullin S, Le Goff B, Marotte H, Mezieres M, Morel J, Perdriger A, Pham T, Pouplin S, Roth O, Ruyssen-Witrand A, Schaeverbeke T, Sellam J, Senbel E, Soubrier M et 10 patients désignés par l'AFP RIC et l'ANDAR. 


\section{Références}

[1] Gaujoux-Viala C, Gossec L, Cantagrel A, Dougados M, Fautrel B, Mariette X, et al. Recommendations of the French Society for Rheumatology for managing rheumatoid arthritis. Jt Bone Spine Rev Rhum 2014;81:287-97.

[2] Smolen JS, Landewé R, Bijlsma J, Burmester G, Chatzidionysiou K, Dougados M, et al. EULAR recommendations for the management of rheumatoid arthritis with synthetic and biological disease-modifying antirheumatic drugs: 2016 update. Ann Rheum Dis 2017;76:960-77.

[3] Combe B, Landewe R, Daien Cl, Hua C, Aletaha D, Álvaro-Gracia JM, et al. 2016 update of the EULAR recommendations for the management of early arthritis. Ann Rheum Dis 2017;76:94859.

[4] Hua C, Daien Cl, Combe B, Landewe R. Diagnosis, prognosis and classification of early arthritis: results of a systematic review informing the 2016 update of the EULAR recommendations for the management of early arthritis. RMD Open 2017;3:e000406.

[5] Daien Cl, Hua C, Combe B, Landewe R. Non-pharmacological and pharmacological interventions in patients with early arthritis: a systematic literature review informing the 2016 update of EULAR recommendations for the management of early arthritis. RMD Open 2017;3:e000404.

[6] Nam JL, Takase-Minegishi K, Ramiro S, Chatzidionysiou K, Smolen JS, van der Heijde D, et al. Efficacy of biological disease-modifying antirheumatic drugs: a systematic literature review informing the 2016 update of the EULAR recommendations for the management of rheumatoid arthritis. Ann Rheum Dis 2017;76:1113-36. doi:10.1136/annrheumdis-2016210713.

[7] Chatzidionysiou K, Emamikia S, Nam J, Ramiro S, Smolen J, van der Heijde D, et al. Efficacy of glucocorticoids, conventional and targeted synthetic disease-modifying antirheumatic drugs: a systematic literature review informing the 2016 update of the EULAR recommendations for the management of rheumatoid arthritis. Ann Rheum Dis 2017;76:1102-7.

[8] Ramiro S, Sepriano A, Chatzidionysiou K, Nam JL, Smolen JS, van der Heijde D, et al. Safety of synthetic and biological DMARDs: a systematic literature review informing the 2016 update of the EULAR recommendations for management of rheumatoid arthritis. Ann Rheum Dis 2017;76:1101-36.

[9] Oxford Centre for Evidence-based Medicine - Levels of Evidence (March 2009). CEBM 2009. http://www.cebm.net/oxford-centre-evidence-based-medicine-levels-evidence-march-2009/ (accessed April 24, 2016).

[10] Beauvais C. L'éducation thérapeutique pour la polyarthrite rhumatoïde : pourquoi, quand et comment ? Rev Rhum Monogr 2018;85:61-5.

[11] Zangi HA, Ndosi M, Adams J, Andersen L, Bode C, Boström C, et al. EULAR recommendations for patient education for people with inflammatory arthritis. Ann Rheum Dis 2015;74:954-62.

[12] Haute Autorité de Santé H. Recommandations. Education thérapeutique du patient. Définitions, finalités et organisation n.d. https://www.hassante.fr/portail/jcms/c_1241714/fr/education-therapeutique-du-patient-etp.

[13] Emery P, Breedveld FC, Dougados M, Kalden JR, Schiff MH, Smolen JS. Early referral recommendation for newly diagnosed rheumatoid arthritis: evidence based development of a clinical guide. Ann Rheum Dis 2002;61:290-7.

[14] Haute Autorité de Santé H. Recommandations professionnelle - Polyarthrite Rhumatoïde Synthèse des recommandations n.d. https://www.hassante.fr/portail/upload/docs/application/pdf/polyarthrite_rhumatoide__synthese_de_lensemble_des_recommandations.pdf.

[15] Husberg M, Davidson T, Hallert E. Non-medical costs during the first year after diagnosis in two cohorts of patients with early rheumatoid arthritis, enrolled 10 years apart. Clin Rheumatol 2017;36:499-506. 
[16] Matsumoto T, Nishino J, Izawa N, Naito M, Hirose J, Tanaka S, et al. Trends in Treatment, Outcomes, and Incidence of Orthopedic Surgery in Patients with Rheumatoid Arthritis: An Observational Cohort Study Using the Japanese National Database of Rheumatic Diseases. J Rheumatol 2017;44:1575-82.

[17] Nystad TW, Fenstad AM, Furnes O, Havelin LI, Skredderstuen AK, Fevang B-T. Reduction in orthopaedic surgery in patients with rheumatoid arthritis: a Norwegian register-based study. Scand J Rheumatol 2016;45:1-7.

[18] ter Wee MM, Lems WF, Usan H, Gulpen A, Boonen A. The effect of biological agents on work participation in rheumatoid arthritis patients: a systematic review. Ann Rheum Dis 2012;71:161-71.

[19] Vanier A, Mariette X, Tubach F, Fautrel B, STRASS Study Group. Cost-Effectiveness of TNFBlocker Injection Spacing for Patients with Established Rheumatoid Arthritis in Remission: An Economic Evaluation from the Spacing of TNF-Blocker Injections in Rheumatoid Arthritis Trial. Value Health J Int Soc Pharmacoeconomics Outcomes Res 2017;20:577-85.

[20] Gulácsi L, Brodszky V, Baji P, Kim H, Kim SY, Cho YY, et al. Biosimilars for the management of rheumatoid arthritis: economic considerations. Expert Rev Clin Immunol 2015;11 Suppl 1:S4352.

[21] van Steenbergen HW, Aletaha D, Beaart-van de Voorde LJ, Brouwer E, Codreanu C, Combe B, et al. EULAR definition of arthralgia suspicious for progression to rheumatoid arthritis. Ann Rheum Dis 2017;76:491-6.

[22] Villeneuve E, Nam JL, Bell MJ, Deighton CM, Felson DT, Hazes JM, et al. A systematic literature review of strategies promoting early referral and reducing delays in the diagnosis and management of inflammatory arthritis. Ann Rheum Dis 2013;72:13-22.

[23] Colebatch AN, Edwards CJ, Østergaard M, van der Heijde D, Balint PV, D'Agostino M-A, et al. EULAR recommendations for the use of imaging of the joints in the clinical management of rheumatoid arthritis. Ann Rheum Dis 2013;72:804-14.

[24] Forien M, Ottaviani S. Ultrasound and follow-up of rheumatoid arthritis. Jt Bone Spine Rev Rhum 2017;84:531-6.

[25] Nam JL, Hensor EMA, Hunt L, Conaghan PG, Wakefield RJ, Emery P. Ultrasound findings predict progression to inflammatory arthritis in anti-CCP antibody-positive patients without clinical synovitis. Ann Rheum Dis 2016;75:2060-7.

[26] Naredo E, Bonilla G, Gamero F, Uson J, Carmona L, Laffon A. Assessment of inflammatory activity in rheumatoid arthritis: a comparative study of clinical evaluation with grey scale and power Doppler ultrasonography. Ann Rheum Dis 2005;64:375-81.

[27] van der Heijde D, van der Helm-van Mil, Aletaha D, Bingham CO, Burmester GR, Dougados M, et al. EULAR definition of erosive disease in light of the 2010 ACR/EULAR rheumatoid arthritis classification criteria. Ann Rheum Dis 2013;72:479-81.

[28] Hua C, Combe B. Les nouveaux critères de classification ACR/EULAR 2010 pour un diagnostic plus précoce de la polyarthrite rhumatoïde. Rev Rhum Monogr 2017;84:337-42.

[29] van Nies JA, Tsonaka R, Gaujoux-Viala C, Fautrel B, van der Helm-van Mil AH. Evaluating relationships between symptom duration and persistence of rheumatoid arthritis: does a window of opportunity exist? Results on the Leiden early arthritis clinic and ESPOIR cohorts. Ann Rheum Dis 2015;74:806-12.

[30] Smolen JS, Breedveld FC, Burmester GR, Bykerk V, Dougados M, Emery P, et al. Treating rheumatoid arthritis to target: 2014 update of the recommendations of an international task force. Ann Rheum Dis 2016;75:3-15.

[31] Scirè CA, Lunt M, Marshall T, Symmons DPM, Verstappen SMM. Early remission is associated with improved survival in patients with inflammatory polyarthritis: results from the Norfolk Arthritis Register. Ann Rheum Dis 2014;73:1677-82.

[32] Dale J, Stirling A, Zhang R, Purves D, Foley J, Sambrook M, et al. Targeting ultrasound remission in early rheumatoid arthritis: the results of the TaSER study, a randomised clinical trial. Ann Rheum Dis 2016;75:1043-50. 
[33] Haavardsholm EA, Aga A-B, Olsen IC, Lillegraven S, Hammer HB, Uhlig T, et al. Ultrasound in management of rheumatoid arthritis: ARCTIC randomised controlled strategy trial. BMJ 2016;354:i4205.

[34] Sepriano A, Ramiro S, Landewe R, van der Heijde D, Ohrndorf S, FitzGerald O, et al. Abstract 2967: Adding Ultrasound to the Treat-to-Target Strategy Shows No Benefit in Achievement of Remission: Results from the Biodam Cohort. Arthritis Rheumatol 2017;Abstract Supplement:4222-3.

[35] Mack ME, Hsia E, Aletaha D. Comparative Assessment of the Different American College of Rheumatology/European League Against Rheumatism Remission Definitions for Rheumatoid Arthritis for Their Use as Clinical Trial End Points. Arthritis Rheumatol Hoboken NJ 2017;69:518-28.

[36] Smolen JS, Aletaha D, Gruben D, Zwillich SH, Krishnaswami S, Mebus C. Brief Report: Remission Rates With Tofacitinib Treatment in Rheumatoid Arthritis: A Comparison of Various Remission Criteria. Arthritis Rheumatol Hoboken NJ 2017;69:728-34.

[37] Fleischmann R, van der Heijde D, Koenig AS, Pedersen R, Szumski A, Marshall L, et al. How much does Disease Activity Score in 28 joints ESR and CRP calculations underestimate disease activity compared with the Simplified Disease Activity Index? Ann Rheum Dis 2015;74:1132-7.

[38] Aletaha D, Smolen JS. Joint damage in rheumatoid arthritis progresses in remission according to the Disease Activity Score in 28 joints and is driven by residual swollen joints. Arthritis Rheum 2011;63:3702-11.

[39] Kavanaugh A, Fleischmann RM, Emery P, Kupper H, Redden L, Guerette B, et al. Clinical, functional and radiographic consequences of achieving stable low disease activity and remission with adalimumab plus methotrexate or methotrexate alone in early rheumatoid arthritis: 26-Week results from the randomised, controlled OPTIMA study. Ann Rheum Dis 2013;72:64-71.

[40] Iking-Konert C, Aringer M, Wollenhaupt J, Mosch T, Tuerk S, Feist E, et al. Performance of the new 2011 ACR/EULAR remission criteria with tocilizumab using the phase Illb study TAMARA as an example and their comparison with traditional remission criteria. Ann Rheum Dis 2011;70:1986-90.

[41] Schipper LG, Vermeer M, Kuper HH, Hoekstra MO, Haagsma CJ, Den Broeder AA, et al. A tight control treatment strategy aiming for remission in early rheumatoid arthritis is more effective than usual care treatment in daily clinical practice: a study of two cohorts in the Dutch Rheumatoid Arthritis Monitoring registry. Ann Rheum Dis 2012;71:845-50.

[42] Schipper LG, van Hulst LTC, Grol R, van Riel PLCM, Hulscher MEJL, Fransen J. Meta-analysis of tight control strategies in rheumatoid arthritis: protocolized treatment has additional value with respect to the clinical outcome. Rheumatol Oxf Engl 2010;49:2154-64.

[43] Landewé R, van der Heijde D, Klareskog L, van Vollenhoven R, Fatenejad S. Disconnect between inflammation and joint destruction after treatment with etanercept plus methotrexate: results from the trial of etanercept and methotrexate with radiographic and patient outcomes. Arthritis Rheum 2006;54:3119-25.

[44] Smolen JS, Han C, van der Heijde DMFM, Emery P, Bathon JM, Keystone E, et al. Radiographic changes in rheumatoid arthritis patients attaining different disease activity states with methotrexate monotherapy and infliximab plus methotrexate: the impacts of remission and tumour necrosis factor blockade. Ann Rheum Dis 2009;68:823-7.

[45] Gaujoux-Viala C, Rincheval N, Dougados M, Combe B, Fautrel B. Optimal methotrexate dose is associated with better clinical outcomes than non-optimal dose in daily practice: results from the ESPOIR early arthritis cohort. Ann Rheum Dis 2017;76:2054-60.

[46] Furst DE, Koehnke R, Burmeister LF, Kohler J, Cargill I. Increasing methotrexate effect with increasing dose in the treatment of resistant rheumatoid arthritis. J Rheumatol 1989;16:31320.

[47] Schiff MH, Sadowski P. Oral to subcutaneous methotrexate dose-conversion strategy in the treatment of rheumatoid arthritis. Rheumatol Int 2017;37:213-8. 
[48] Visser K, Katchamart W, Loza E, Martinez-Lopez JA, Salliot C, Trudeau J, et al. Multinational evidence-based recommendations for the use of methotrexate in rheumatic disorders with a focus on rheumatoid arthritis: integrating systematic literature research and expert opinion of a broad international panel of rheumatologists in the $3 E$ Initiative. Ann Rheum Dis 2009;68:1086-93.

[49] Verschueren P, De Cock D, Corluy L, Joos R, Langenaken C, Taelman V, et al. Methotrexate in combination with other DMARDs is not superior to methotrexate alone for remission induction with moderate-to-high-dose glucocorticoid bridging in early rheumatoid arthritis after 16 weeks of treatment: the CareRA trial. Ann Rheum Dis 2015;74:27-34.

[50] ter Wee MM, den Uyl D, Boers M, Kerstens P, Nurmohamed M, van Schaardenburg D, et al. Intensive combination treatment regimens, including prednisolone, are effective in treating patients with early rheumatoid arthritis regardless of additional etanercept: 1-year results of the COBRA-light open-label, randomised, non-inferiority trial. Ann Rheum Dis 2015;74:123340.

[51] De Jong PH, Hazes JM, Han HK, Huisman M, Van Zeben D, Van Der Lubbe PA, et al. Randomised comparison of initial triple DMARD therapy with methotrexate monotherapy in combination with low-dose glucocorticoid bridging therapy; 1-year data of the TREACH trial. Ann Rheum Dis 2014;73:1331-9.

[52] Takeuchi T, Yamanaka H, Ishiguro N, Miyasaka N, Mukai M, Matsubara T, et al. Adalimumab, a human anti-TNF monoclonal antibody, outcome study for the prevention of joint damage in Japanese patients with early rheumatoid arthritis: The HOPEFUL 1 study. Ann Rheum Dis 2014;73:536-43.

[53] Scott IC, Ibrahim F, Simpson G, Kowalczyk A, White-Alao B, Hassell A, et al. A randomised trial evaluating anakinra in early active rheumatoid arthritis. Clin Exp Rheumatol 2016;34:88-93.

[54] Atsumi T, Yamamoto K, Takeuchi T, Yamanaka H, Ishiguro N, Tanaka Y, et al. The first doubleblind, randomised, parallel-group certolizumab pegol study in methotrexate-naive early rheumatoid arthritis patients with poor prognostic factors, C-OPERA, shows inhibition of radiographic progression. Ann Rheum Dis 2016;75:75-83.

[55] Bijlsma JWJ, Welsing PMJ, Woodworth TG, Middelink LM, Pethö-Schramm A, Bernasconi C, et al. Early rheumatoid arthritis treated with tocilizumab, methotrexate, or their combination (UAct-Early): a multicentre, randomised, double-blind, double-dummy, strategy trial. Lancet Lond Engl 2016;388:343-55.

[56] Nam JL, Villeneuve E, Hensor EM, Conaghan PG, Keen HI, Buch MH, et al. Remission induction comparing infliximab and high-dose intravenous steroid, followed by treat-to-target: a doubleblind, randomised, controlled trial in new-onset, treatment-naive, rheumatoid arthritis (the IDEA study). Ann Rheum Dis 2014;73:75-85.

[57] Rempenault C, Combe B, Barnetche T, Gaujoux-Viala C, Lukas C, Morel J, et al. Metabolic and cardiovascular benefits of hydroxychloroquine in patients with rheumatoid arthritis: a systematic review and meta-analysis. Ann Rheum Dis 2018;77:98-103.

[58] Verschueren P, De Cock D, Corluy L, Joos R, Langenaken C, Taelman V, et al. Effectiveness of methotrexate with step-down glucocorticoid remission induction (COBRA Slim) versus other intensive treatment strategies for early rheumatoid arthritis in a treat-to-target approach: 1year results of CareRA, a randomised pragmatic open-label superiority trial. Ann Rheum Dis 2017;76:511-20.

[59] Safy M, Jacobs J, IJff ND, Bijlsma J, van Laar JM, de Hair M, et al. Long-term outcome is better when a methotrexate-based treatment strategy is combined with $10 \mathrm{mg}$ prednisone daily: follow-up after the second Computer-Assisted Management in Early Rheumatoid Arthritis trial. Ann Rheum Dis 2017;76:1432-5.

[60] Roubille C, Rincheval N, Dougados M, Flipo R-M, Daurès J-P, Combe B. Seven-year tolerability profile of glucocorticoids use in early rheumatoid arthritis: data from the ESPOIR cohort. Ann Rheum Dis 2017;76:1797-802. 
[61] Ruyssen-Witrand A, Constantin A. Controversies in rheumatoid arthritis glucocorticoid therapy. Jt Bone Spine Rev Rhum 2018;85:417-22.

[62] Listing J, Kekow J, Manger B, Burmester G-R, Pattloch D, Zink A, et al. Mortality in rheumatoid arthritis: the impact of disease activity, treatment with glucocorticoids, TNF $\alpha$ inhibitors and rituximab. Ann Rheum Dis 2015;74:415-21.

[63] Roubille C, Richer V, Starnino T, McCourt C, McFarlane A, Fleming P, et al. The effects of tumour necrosis factor inhibitors, methotrexate, non-steroidal anti-inflammatory drugs and corticosteroids on cardiovascular events in rheumatoid arthritis, psoriasis and psoriatic arthritis: a systematic review and meta-analysis. Ann Rheum Dis 2015;74:480-9.

[64] de Jong PH, Hazes JM, Han HK, Huisman M, van Zeben D, van der Lubbe PA, et al. Randomised comparison of initial triple DMARD therapy with methotrexate monotherapy in combination with low-dose glucocorticoid bridging therapy; 1-year data of the tREACH trial. Ann Rheum Dis 2014;73:1331-9.

[65] Nam JL, Villeneuve E, Hensor EM, Conaghan PG, Keen HI, Buch MH, et al. Remission induction comparing infliximab and high-dose intravenous steroid, followed by treat-to-target: a doubleblind, randomised, controlled trial in new-onset, treatment-naive, rheumatoid arthritis (the IDEA study). Ann Rheum Dis 2014;73:75-85.

[66] Fautrel B, Granger B, Combe B, Saraux A, Guillemin F, Le Loet X. Matrix to predict rapid radiographic progression of early rheumatoid arthritis patients from the community treated with methotrexate or leflunomide: Results from the ESPOIR cohort. Arthritis Res Ther 2012;14:R249.

[67] Vastesaeger N, Xu S, Aletaha D, St Clair EW, Smolen JS. A pilot risk model for the prediction of rapid radiographic progression in rheumatoid arthritis. Rheumatol Oxf Engl 2009;48:1114-21.

[68] Klarenbeek NB, Güler-Yüksel M, van der Kooij SM, Han KH, Ronday HK, Kerstens PJSM, et al. The impact of four dynamic, goal-steered treatment strategies on the 5-year outcomes of rheumatoid arthritis patients in the BeSt study. Ann Rheum Dis 2011;70:1039-46.

[69] Kiely P, Walsh D, Williams R, Young A, Early Rheumatoid Arthritis N. Outcome in rheumatoid arthritis patients with continued conventional therapy for moderate disease activity--the early RA network (ERAN). Rheumatology 2011;50:926-31.

[70] Fleischmann R, Mysler E, Hall S, Kivitz AJ, Moots RJ, Luo Z, et al. Efficacy and safety of tofacitinib monotherapy, tofacitinib with methotrexate, and adalimumab with methotrexate in patients with rheumatoid arthritis (ORAL Strategy): a phase 3b/4, double-blind, head-tohead, randomised controlled trial. Lancet Lond Engl 2017;390:457-68.

[71] Schiff $M$, Weinblatt ME, Valente R, van der Heijde D, Citera G, Elegbe A, et al. Head-to-head comparison of subcutaneous abatacept versus adalimumab for rheumatoid arthritis: two-year efficacy and safety findings from AMPLE trial. Ann Rheum Dis 2014;73:86-94.

[72] Taylor PC, Keystone EC, van der Heijde D, Weinblatt ME, Del Carmen Morales L, Reyes Gonzaga J, et al. Baricitinib versus Placebo or Adalimumab in Rheumatoid Arthritis. N Engl J Med 2017;376:652-62.

[73] Nam JL, Takase-Minegishi K, Ramiro S, Chatzidionysiou K, Smolen JS, van der Heijde D, et al. Efficacy of biological disease-modifying antirheumatic drugs: a systematic literature review informing the 2016 update of the EULAR recommendations for the management of rheumatoid arthritis. Ann Rheum Dis 2017;76:1113-36.

[74] Singh JA, Hossain A, Tanjong Ghogomu E, Mudano AS, Tugwell P, Wells GA. Biologic or tofacitinib monotherapy for rheumatoid arthritis in people with traditional disease-modifying anti-rheumatic drug (DMARD) failure: a Cochrane Systematic Review and network metaanalysis (NMA). Cochrane Database Syst Rev 2016;11:CD012437.

[75] de La Forest Divonne M, Gottenberg JE, Salliot C. Safety of biologic DMARDs in RA patients in real life: A systematic literature review and meta-analyses of biologic registers. Jt Bone Spine Rev Rhum 2017;84:133-40. 
[76] Cohen SB, Tanaka Y, Mariette X, Curtis JR, Lee EB, Nash P, et al. Long-term safety of tofacitinib for the treatment of rheumatoid arthritis up to 8.5 years: integrated analysis of data from the global clinical trials. Ann Rheum Dis 2017;76:1253-62.

[77] Curtis JR, Xie F, Yun H, Bernatsky S, Winthrop KL. Real-world comparative risks of herpes virus infections in tofacitinib and biologic-treated patients with rheumatoid arthritis. Ann Rheum Dis 2016;75:1843-7.

[78] Hauser SL, Waubant E, Arnold DL, Vollmer T, Antel J, Fox RJ, et al. B-cell depletion with rituximab in relapsing-remitting multiple sclerosis. N Engl J Med 2008;358:676-88.

[79] Coleman M, Lammers PE, Ciceri F, Jacobs IA. Role of Rituximab and Rituximab Biosimilars in Diffuse Large B-Cell Lymphoma. Clin Lymphoma Myeloma Leuk 2016;16:175-81.

[80] Khoury SJ, Rochon J, Ding L, Byron M, Ryker K, Tosta P, et al. ACCLAIM: A randomized trial of abatacept (CTLA4-Ig) for relapsing-remitting multiple sclerosis. Mult Scler Houndmills Basingstoke Engl 2017;23:686-95.

[81] Scott DL, Ibrahim F, Farewell V, O'Keeffe AG, Walker D, Kelly C, et al. Tumour necrosis factor inhibitors versus combination intensive therapy with conventional disease modifying antirheumatic drugs in established rheumatoid arthritis: TACIT non-inferiority randomised controlled trial. BMJ 2015;350:h1046.

[82] Fleischmann R, Tongbram V, van Vollenhoven R, Tang DH, Chung J, Collier D, et al. Systematic review and network meta-analysis of the efficacy and safety of tumour necrosis factor inhibitor-methotrexate combination therapy versus triple therapy in rheumatoid arthritis. RMD Open 2017;3:e000371.

[83] Buckley F, Finckh A, Huizinga TWJ, Dejonckheere F, Jansen JP. Comparative Efficacy of Novel DMARDs as Monotherapy and in Combination with Methotrexate in Rheumatoid Arthritis Patients with Inadequate Response to Conventional DMARDs: A Network Meta-Analysis. J Manag Care Spec Pharm 2015;21:409-23.

[84] Dougados M, Kissel K, Sheeran T, Tak PP, Conaghan PG, Mola EM, et al. Adding tocilizumab or switching to tocilizumab monotherapy in methotrexate inadequate responders: 24-week symptomatic and structural results of a 2-year randomised controlled strategy trial in rheumatoid arthritis (ACT-RAY). Ann Rheum Dis 2013;72:43-50.

[85] Burmester GR, Rigby WF, van Vollenhoven RF, Kay J, Rubbert-Roth A, Blanco R, et al. Tocilizumab combination therapy or monotherapy or methotrexate monotherapy in methotrexate-naive patients with early rheumatoid arthritis: 2-year clinical and radiographic results from the randomised, placebo-controlled FUNCTION trial. Ann Rheum Dis 2017;76:1279-84.

[86] Kaneko Y, Atsumi T, Tanaka Y, Inoo M, Kobayashi-Haraoka H, Amano K, et al. Comparison of adding tocilizumab to methotrexate with switching to tocilizumab in patients with rheumatoid arthritis with inadequate response to methotrexate: 52-week results from a prospective, randomised, controlled study (SURPRISE study). Ann Rheum Dis 2016;75:1917-23.

[87] Fleischmann R, Schiff M, van der Heijde D, Ramos-Remus C, Spindler A, Stanislav M, et al. Baricitinib, Methotrexate, or Combination in Patients With Rheumatoid Arthritis and No or Limited Prior Disease-Modifying Antirheumatic Drug Treatment. Arthritis Rheumatol Hoboken NJ 2017;69:506-17.

[88] Burmester G-R, Kivitz AJ, Kupper H, Arulmani U, Florentinus S, Goss SL, et al. Efficacy and safety of ascending methotrexate dose in combination with adalimumab: the randomised CONCERTO trial. Ann Rheum Dis 2015;74:1037-44.

[89] Kaeley GS, Evangelisto AM, Nishio MJ, Goss SL, Liu S, Kalabic J, et al. Methotrexate Dosage Reduction Upon Adalimumab Initiation: Clinical and Ultrasonographic Outcomes from the Randomized Noninferiority MUSICA Trial. J Rheumatol 2016;43:1480-9.

[90] Finckh A, Dehler S, Gabay C, SCQM doctors. The effectiveness of leflunomide as a co-therapy of tumour necrosis factor inhibitors in rheumatoid arthritis: a population-based study. Ann Rheum Dis 2009;68:33-9. 
[91] De Stefano R, Frati E, Nargi F, Baldi C, Menza L, Hammoud M, et al. Comparison of combination therapies in the treatment of rheumatoid arthritis: leflunomide-anti-TNF-alpha versus methotrexate-anti-TNF-alpha. Clin Rheumatol 2010;29:517-24.

[92] Soliman MM, Hyrich KL, Lunt M, Watson KD, Symmons DPM, Ashcroft DM, et al. Effectiveness of rituximab in patients with rheumatoid arthritis: observational study from the British Society for Rheumatology Biologics Register. J Rheumatol 2012;39:240-6.

[93] Schaeverbeke T, Truchetet M-E, Kostine M, Barnetche T, Bannwarth B, Richez C. Immunogenicity of biologic agents in rheumatoid arthritis patients: lessons for clinical practice. Rheumatol Oxf Engl 2016;55:210-20.

[94] Gabay C, Riek M, Hetland ML, Hauge E-M, Pavelka K, Tomšič M, et al. Effectiveness of tocilizumab with and without synthetic disease-modifying antirheumatic drugs in rheumatoid arthritis: results from a European collaborative study. Ann Rheum Dis 2016;75:1336-42.

[95] Lauper K, Nordström D, Pavelka K. Retention of tocilizumab as monotherapy versus TNF inhibitors with conventional synthetic DMARDs in rheumatoid arthritis patients with inadequate response to TNF inhibitors: a study from the TOCERRA collaboration, Ann Rheum Dis; 2017;76(suppl 2):850.

[96] Gabay C, Emery P, van Vollenhoven R, Dikranian A, Alten R, Pavelka K, et al. Tocilizumab monotherapy versus adalimumab monotherapy for treatment of rheumatoid arthritis (ADACTA): a randomised, double-blind, controlled phase 4 trial. Lancet Lond Engl 2013;381:1541-50.

[97] Burmester GR, Lin Y, Patel R, van Adelsberg J, Mangan EK, Graham NMH, et al. Efficacy and safety of sarilumab monotherapy versus adalimumab monotherapy for the treatment of patients with active rheumatoid arthritis (MONARCH): a randomised, double-blind, parallelgroup phase III trial. Ann Rheum Dis 2017;76:840-7.

[98] van Riel PLCM, Taggart AJ, Sany J, Gaubitz M, Nab HW, Pedersen R, et al. Efficacy and safety of combination etanercept and methotrexate versus etanercept alone in patients with rheumatoid arthritis with an inadequate response to methotrexate: the ADORE study. Ann Rheum Dis 2006;65:1478-83.

[99] Lethaby A, Lopez-Olivo MA, Maxwell L, Burls A, Tugwell P, Wells GA. Etanercept for the treatment of rheumatoid arthritis. Cochrane Database Syst Rev 2013:CD004525.

[100] Truchetet M-E, Poursac N, Barnetche T, Shipley E, Gottenberg J-E, Bannwarth B, et al. Abatacept monotherapy compared with abatacept plus disease-modifying anti-rheumatic drugs in rheumatoid arthritis patients: data from the ORA registry. Arthritis Res Ther 2016;18:72.

[101] Nüßlein HG, Alten R, Galeazzi M, Lorenz H-M, Nurmohamed MT, Bensen WG, et al. Prognostic factors for abatacept retention in patients who received at least one prior biologic agent: an interim analysis from the observational, prospective ACTION study. BMC Musculoskelet Disord 2015;16:176.

[102] Schoels M, Aletaha D, Smolen JS, Wong JB. Comparative effectiveness and safety of biological treatment options after tumour necrosis factor $\alpha$ inhibitor failure in rheumatoid arthritis: systematic review and indirect pairwise meta-analysis. Ann Rheum Dis 2012;71:1303-8.

[103] Manders SHM, Kievit W, Adang E, Brus HL, Moens HJB, Hartkamp A, et al. Cost-effectiveness of abatacept, rituximab, and TNFi treatment after previous failure with TNFi treatment in rheumatoid arthritis: a pragmatic multi-centre randomised trial. Arthritis Res Ther 2015;17:134.

[104] Gottenberg J-E, Brocq O, Perdriger A, Lassoued S, Berthelot J-M, Wendling D, et al. Non-TNFTargeted Biologic vs a Second Anti-TNF Drug to Treat Rheumatoid Arthritis in Patients With Insufficient Response to a First Anti-TNF Drug: A Randomized Clinical Trial. JAMA 2016;316:1172-80.

[105] Du Pan SM, Scherer A, Gabay C, Finckh A. Differential drug retention between anti-TNF agents and alternative biological agents after inadequate response to an anti-TNF agent in rheumatoid arthritis patients. Ann Rheum Dis 2012;71:997-9. 
[106] Smolen JS, Burmester G-R, Combe B, Curtis JR, Hall S, Haraoui B, et al. Head-to-head comparison of certolizumab pegol versus adalimumab in rheumatoid arthritis: 2-year efficacy and safety results from the randomised EXXELERATE study. Lancet Lond Engl 2016;388:276374.

[107] Curtis JR, Bykerk VP, Aassi M, Schiff M. Adherence and Persistence with Methotrexate in Rheumatoid Arthritis: A Systematic Review. J Rheumatol 2016;43:1997-2009.

[108] de Thurah A, Nørgaard M, Harder I, Stengaard-Pedersen K. Compliance with methotrexate treatment in patients with rheumatoid arthritis: influence of patients' beliefs about the medicine. A prospective cohort study. Rheumatol Int 2010;30:1441-8.

[109] Lillegraven S, Prince FHM, Shadick NA, Bykerk VP, Lu B, Frits ML, et al. Remission and radiographic outcome in rheumatoid arthritis: application of the 2011 ACR/EULAR remission criteria in an observational cohort. Ann Rheum Dis 2012;71:681-6.

[110] Sigaux J, Bailly F, Hajage D, Mariette X, Morel J, Gandjbakhch F, et al. Sustainability of TNFblocker tapering in rheumatoid arthritis over 3 years: long-term follow-up of the STRASS (Spacing of TNF-blocker injections in Rheumatoid ArthritiS Study) randomised controlled trial. RMD Open 2017;3:e000474.

[111] Bouman CA, van Herwaarden N, van den Hoogen FH, Fransen J, van Vollenhoven RF, Bijlsma JW, et al. Long-term outcomes after disease activity-guided dose reduction of TNF inhibition in rheumatoid arthritis: 3-year data of the DRESS study - a randomised controlled pragmatic noninferiority strategy trial. Ann Rheum Dis 2017;76:1716-22.

[112] Takeuchi T, Matsubara T, Ohta S, Mukai M, Amano K, Tohma S, et al. Biologic-free remission of established rheumatoid arthritis after discontinuation of abatacept: a prospective, multicentre, observational study in Japan. Rheumatol Oxf Engl 2015;54:683-91.

[113] Mariette X, Rouanet S, Sibilia J, Combe B, Le Loët X, Tebib J, et al. Evaluation of low-dose rituximab for the retreatment of patients with active rheumatoid arthritis: a non-inferiority randomised controlled trial. Ann Rheum Dis 2014;73:1508-14.

[114] Takeuchi T, Genovese MC, Haraoui B, Li Z, Xie L, Klar R. Abstract 1821: Dose Reduction of Baricitinib in Patients with Rheumatoid Arthritis Achieving Sustained Disease Control: Results of a Prospective Stud. Arthritis Rheumatol 2017;Abstract Supplement:2594-6.

[115] Smolen JS, Emery P, Ferraccioli GF, Samborski W, Berenbaum F, Davies OR, et al. Certolizumab pegol in rheumatoid arthritis patients with low to moderate activity: the CERTAIN doubleblind, randomised, placebo-controlled trial. Ann Rheum Dis 2015;74:843-50.

[116] Schett G, Emery P, Tanaka Y, Burmester G, Pisetsky DS, Naredo E, et al. Tapering biologic and conventional DMARD therapy in rheumatoid arthritis: current evidence and future directions. Ann Rheum Dis 2016;75:1428-37.

[117] Fautrel B, Den Broeder AA. De-intensifying treatment in established rheumatoid arthritis (RA): Why, how, when and in whom can DMARDs be tapered? Best Pract Res Clin Rheumatol 2015;29:550-65.

[118] Kuijper TM, Luime JJ, de Jong PHP, Gerards AH, van Zeben D, Tchetverikov I, et al. Tapering conventional synthetic DMARDs in patients with early arthritis in sustained remission: 2-year follow-up of the tREACH trial. Ann Rheum Dis 2016;75:2119-23.

[119] Haschka J, Englbrecht M, Hueber AJ, Manger B, Kleyer A, Reiser M, et al. Relapse rates in patients with rheumatoid arthritis in stable remission tapering or stopping antirheumatic therapy: interim results from the prospective randomised controlled RETRO study. Ann Rheum Dis 2016;75:45-51.

[120] Radner H, Yoshida K, Frits M, lannaccone C, Shadick NA, Weinblatt M, et al. The impact of multimorbidity status on treatment response in rheumatoid arthritis patients initiating disease-modifying anti-rheumatic drugs. Rheumatol Oxf Engl 2015;54:2076-84.

[121] Peters MJL, van Halm VP, Voskuyl AE, Smulders YM, Boers M, Lems WF, et al. Does rheumatoid arthritis equal diabetes mellitus as an independent risk factor for cardiovascular disease? A prospective study. Arthritis Rheum 2009;61:1571-9. 
[122] Roubille C, Richer V, Starnino T, McCourt C, McFarlane A, Fleming P, et al. The effects of tumour necrosis factor inhibitors, methotrexate, non-steroidal anti-inflammatory drugs and corticosteroids on cardiovascular events in rheumatoid arthritis, psoriasis and psoriatic arthritis: a systematic review and meta-analysis. Ann Rheum Dis 2015;74:480-9.

[123] Agca R, Heslinga SC, Rollefstad S, Heslinga M, Mclnnes IB, Peters MJL, et al. EULAR recommendations for cardiovascular disease risk management in patients with rheumatoid arthritis and other forms of inflammatory joint disorders: 2015/2016 update. Ann Rheum Dis 2017;76:17-28.

[124] Daïen Cl, Tubery A, Cailar G du, Mura T, Roubille F, Morel J, et al. Application of the 2015/2016 EULAR recommendations for cardiovascular risk in daily practice: data from an observational study. Ann Rheum Dis 2018;77:625-6.

[125] Baillet A, Gossec L, Carmona L, Wit M de, van Eijk-Hustings $Y$, Bertheussen $H$, et al. Points to consider for reporting, screening for and preventing selected comorbidities in chronic inflammatory rheumatic diseases in daily practice: a EULAR initiative. Ann Rheum Dis 2016;75:965-73.

[126] van Assen S, Agmon-Levin N, Elkayam O, Cervera R, Doran MF, Dougados M, et al. EULAR recommendations for vaccination in adult patients with autoimmune inflammatory rheumatic diseases. Ann Rheum Dis 2011;70:414-22.

[127] Mikuls TR, Payne JB, Yu F, Thiele GM, Reynolds RJ, Cannon GW, et al. Periodontitis and Porphyromonas gingivalis in patients with rheumatoid arthritis. Arthritis Rheumatol Hoboken NJ 2014;66:1090-100.

[128] Chou Y-Y, Lai K-L, Chen D-Y, Lin C-H, Chen H-H. Rheumatoid Arthritis Risk Associated with Periodontitis Exposure: A Nationwide, Population-Based Cohort Study. PloS One 2015;10:e0139693.

[129] Ortiz P, Bissada NF, Palomo L, Han YW, Al-Zahrani MS, Panneerselvam A, et al. Periodontal therapy reduces the severity of active rheumatoid arthritis in patients treated with or without tumor necrosis factor inhibitors. J Periodontol 2009;80:535-40.

[130] Daïen Cl, Tubery A, Beurai-Weber M, Cailar G du, Picot M-C, Roubille F, et al. Relevance and feasibility of a systematic screening of multimorbidities in patients with chronic inflammatory rheumatic diseases. Joint Bone Spine 2018. doi:10.1016/j.jbspin.2018.03.016.

[131] Dougados M, Soubrier M, Perrodeau E, Gossec L, Fayet F, Gilson M, et al. Impact of a nurseled programme on comorbidity management and impact of a patient self-assessment of disease activity on the management of rheumatoid arthritis: results of a prospective, multicentre, randomised, controlled trial (COMEDRA). Ann Rheum Dis 2015;74:1725-33.

[132] Gossec L, Baillet A, Dadoun S, Daien C, Berenbaum F, Dernis E, et al. Collection and management of selected comorbidities and their risk factors in chronic inflammatory rheumatic diseases in daily practice in France. Jt Bone Spine Rev Rhum 2016;83:501-9.

[133] Sisti LG, Dajko M, Campanella P, Shkurti E, Ricciardi W, de Waure C. The effect of multifactorial lifestyle interventions on cardiovascular risk factors: a systematic review and meta-analysis of trials conducted in the general population and high risk groups. Prev Med 2018;109:82-97.

[134] Thomsen T, Aadahl M, Beyer N, Hetland ML, Løppenthin K, Midtgaard J, et al. The efficacy of motivational counselling and SMS reminders on daily sitting time in patients with rheumatoid arthritis: a randomised controlled trial. Ann Rheum Dis 2017;76:1603-6.

[135] Joseph RM, Movahedi M, Dixon WG, Symmons DPM. Smoking-Related Mortality in Patients With Early Rheumatoid Arthritis: A Retrospective Cohort Study Using the Clinical Practice Research Datalink. Arthritis Care Res 2016;68:1598-606.

[136] Soderlin MK, Petersson IF, Bergman S, Svensson B, group B study. Smoking at onset of rheumatoid arthritis (RA) and its effect on disease activity and functional status: experiences from BARFOT, a long-term observational study on early RA. Scand J Rheumatol 2011;40:24955. 
[137] Vittecoq O, Richard L, Banse C, Lequerré T. The impact of smoking on rheumatoid arthritis outcomes. Jt Bone Spine Rev Rhum 2018;85:135-8.

[138] Guasch-Ferré M, Hu FB, Martínez-González MA, Fitó M, Bulló M, Estruch R, et al. Olive oil intake and risk of cardiovascular disease and mortality in the PREDIMED Study. BMC Med 2014;12:78.

[139] McKellar G, Morrison E, McEntegart A, Hampson R, Tierney A, Mackle G, et al. A pilot study of a Mediterranean-type diet intervention in female patients with rheumatoid arthritis living in areas of social deprivation in Glasgow. Ann Rheum Dis 2007;66:1239-43.

[140] Gioxari A, Kaliora AC, Marantidou F, Panagiotakos DP. Intake of $\omega-3$ polyunsaturated fatty acids in patients with rheumatoid arthritis: A systematic review and meta-analysis. Nutr Burbank Los Angel Cty Calif 2018;45:114-124.e4.

[141] Potter MDE, Brienesse SC, Walker MM, Boyle A, Talley NJ. Effect of the gluten-free diet on cardiovascular risk factors in patients with coeliac disease: A systematic review. J Gastroenterol Hepatol 2018;33:781-91.

[142] Lebwohl B, Cao Y, Zong G, Hu FB, Green PHR, Neugut Al, et al. Long term gluten consumption in adults without celiac disease and risk of coronary heart disease: prospective cohort study. BMJ 2017;357:j1892. 


\section{LEGENDES DES FIGURES}

Figure 1. Nomenclature des traitements de fond. Parmi les traitements biologiques, on retrouve abatacept, adalimumab, certolizumab pegol, etanercept, golimumab, infliximab, rituximab, sarilumab, tocilizumab, etc. bo: biological originator; bs: biosimilar; cs : conventional synthetic; DMARD : disease-modifying anti-rheumatic drug; ts : targeted synthetic

Figure 2. Objectifs du traitement et organisation de la prise en charge. CRP : protéine Créactive ; CDAI : Clinical Disease Activity Index ; DAS28 : disease activity score ; HAQ : Health Assessment Questionnaire; NAG : nombre d'articulations gonflées ; NAD : nombre d'articulations douloureuses; PR : polyarthrite rhumatoïde; SDAI : Simplified Disease Activity Index. Figure adaptée de Smolen et al. [2]

Figure 3. Stratégie de prise en charge médicamenteuse de la PR. ACPA : anticorps antipeptides citrullinées; bDMARD : traitement de fond biologique ; CI : contre-indication ; csDMARD : traitement de fond conventionnel synthétique ; FR : facteur rhumatoïde ; IL-6R : récepteur de l'interleukine 6; LEF: leflunomide; MTX: méthotrexate; reco : recommandations ; SSZ : sulfasalazine ; TNF : tumor necrosis factor ; tsDMARD : traitement de fond synthétique ciblé.

Figure 4. Prise en charge globale des patients atteints de polyarthrite rhumatoïde (PR). Vaccins DTP (diphtérie-tétanos-poliomyélite) tous les 10 ans, grippe annuelle, pneumocoque tous les 5 ans. Le reste du calendrier vaccinal est identique à celui de la population générale. 


\section{TRAITEMENTS DE FOND}

CONVENTIONNELS SYNTHÉTIQUES :

csDMARDs (méthotrexate, leflunomide, sulfasalazine, hydroxychloroquine)

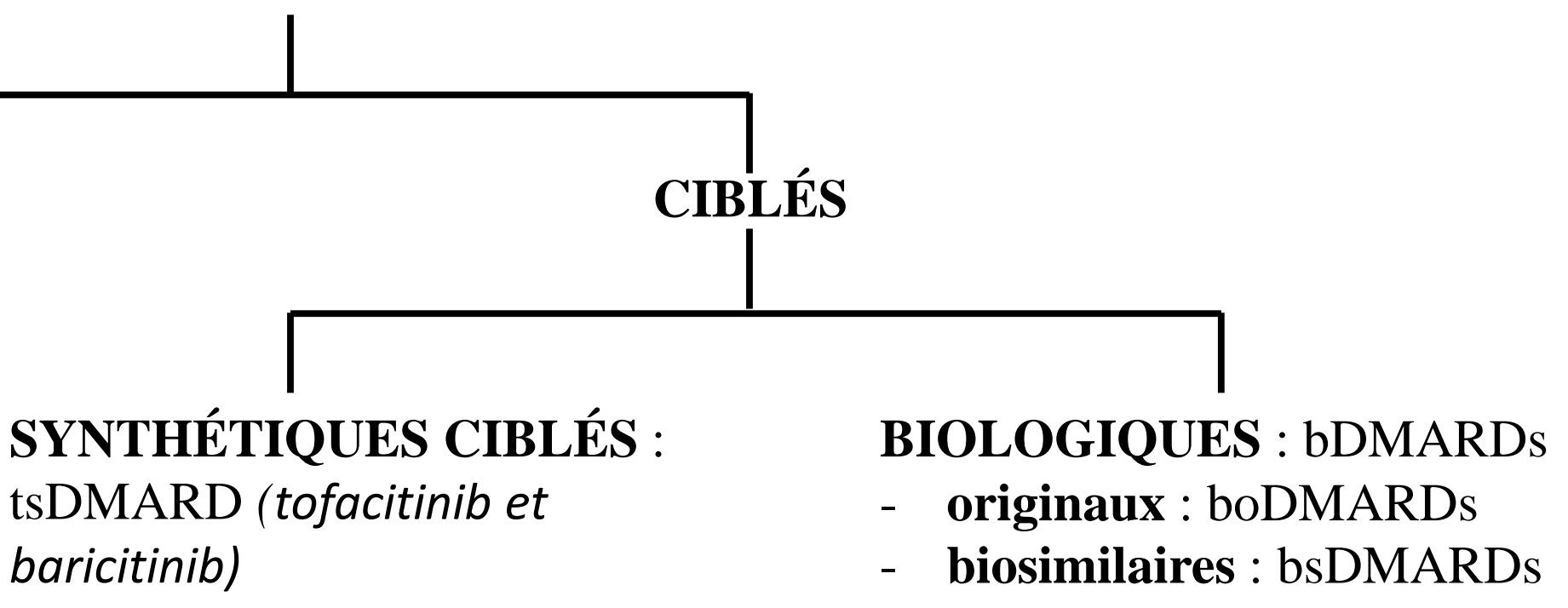




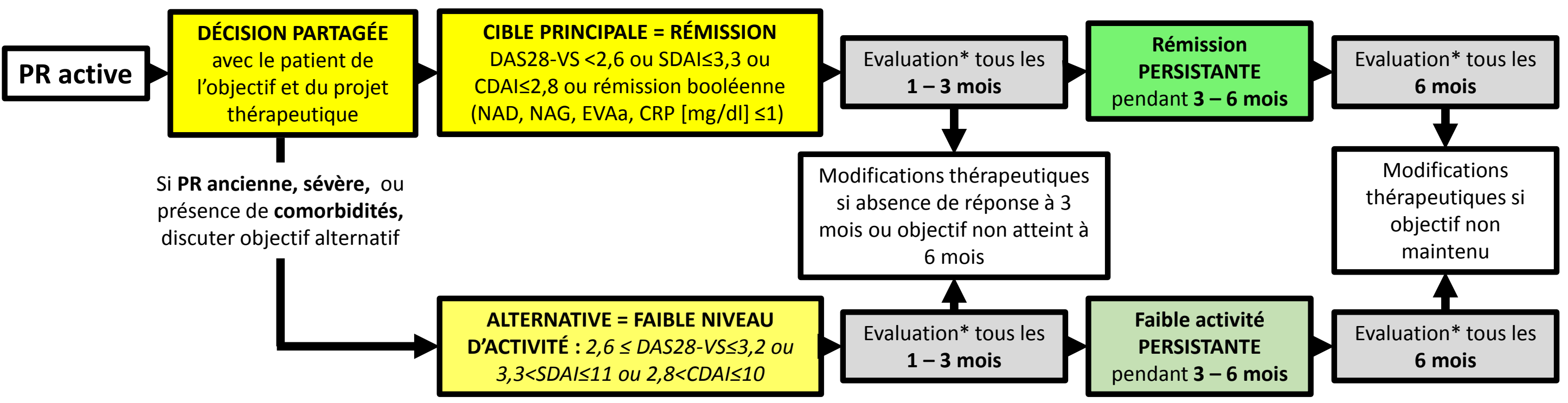

* Evaluation de l'efficacité basée sur la réalisation de l'objectif. Evaluation du retentissement fonctionnel par le HAQ recommandé de façon annuelle. Suivi radiographique recommandé, surtout en cas d'activité clinique résiduelle et en cas de changement de traitement de fond. 
MÉTHOTREXATE (MTX) : posologie optimale (15$30 \mathrm{mg} / \mathrm{S}$ ) en $4-8 \mathrm{~S}$

$\pm$

Corticoïdes : dose cumulée faible, 6 mois max

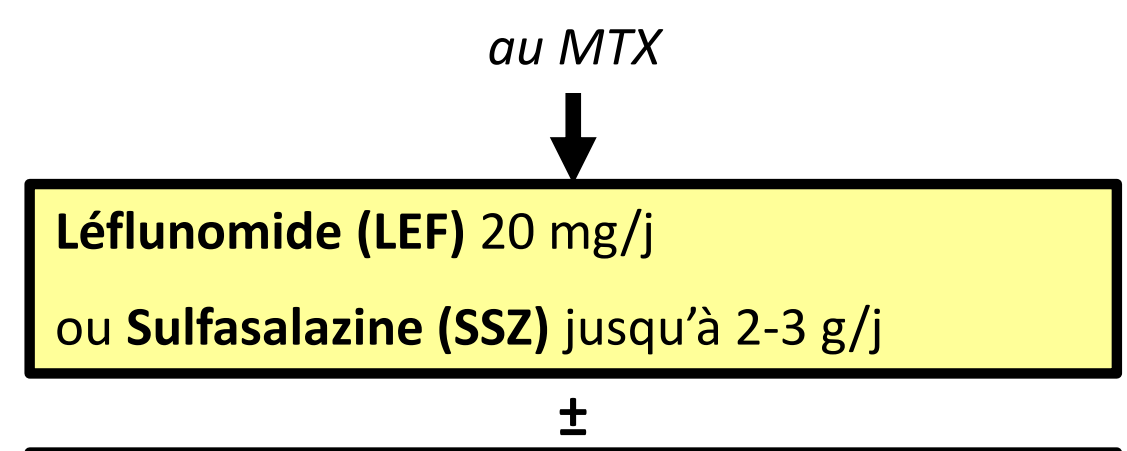

Corticoïdes : dose cumulée faible, 6 mois max

Amélioration significative à 3 mois et atteinte de l'objectif à 6 mois ?

AJOUT D'UNE THÉRAPIE CIBLÉE

bDMARDs (anti-TNF, anti-IL-6R, abatacept et en cas de $\mathrm{Cl}$, rituximab)

ou tsDMARD (JAKi)
Amélioration significative à 3 mois et atteinte de l'objectif à 6 mois ?

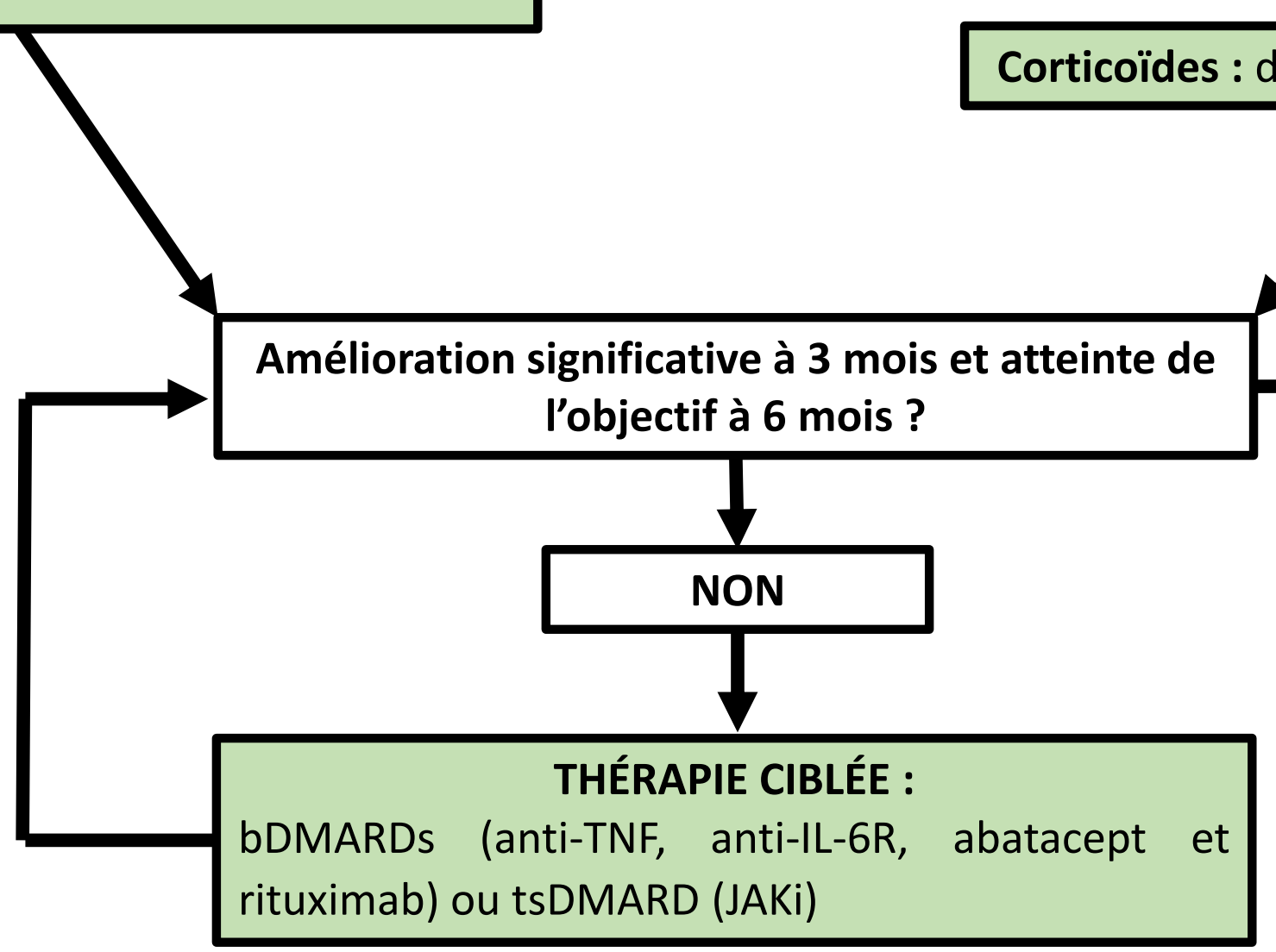

OUI : poursuivre le traitement puis, si rémission prolongée sans corticoïde, discuter la décroissance du csDMARD
CHANGEMENT OU AJOUT D'UN AUTRE

csDMARD : LEF, SSZ, MTX seuls ou en combinaison

$\pm$

FACTEURS DE MAUVAIS PRONOSTIC?

Érosions, FR / ACPA, activité modérée

à forte, échec de $\geq 2$ csDMARDs

\section{NON}


Prise en charge globale et pluridisciplinaire

RHUMATOLOGUE, MEDECIN TRAITANT, kinésithérapeute/ergothérapeute, infirmière spécialisée, cardiologue, diététicien, dentiste, psychologue, assistant social

\section{INFORMATION du patient EDUCATION THÉRAPEUTIQUE DÉCISION MÉDICALE PARTAGÉE}

Suivi coordonné avec le MÉDECIN TRAITANT

ACTIVITÉ PHYSIQUE

Rééducation fonctionnelle

\pm Orthèses et chirurgie

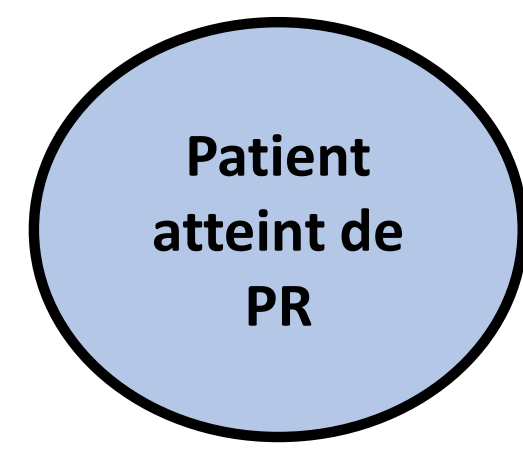

Soutien PSYCHOLOGIQUE

Accompagnement

SOCIOPROFESSIONNEL

Alimentation équilibrée

Eviction des régimes

restrictifs à risque

\section{EVALUATION RÉGULIÈRE \pm PRISE EN CHARGE :}

des facteurs de risque $\mathrm{CV}$ : bilan lipidique (hors poussée), pression artérielle, tabagisme, obésité

du statut vaccinal (grippe, DTP, pneumocoque) et dépistages néoplasiques

de l'état buccodentaire

du risque d'ostéoporose

de l'atteinte pulmonaire, digestive

de la dépression 
Tableau 1. Définitions et nomenclature.

\begin{tabular}{ll}
\hline Terminologie & Définition \\
\hline Facteurs de mauvais pronostic & Erosions osseuses précoces \\
& - Niveau élevé de syndrome inflammatoire \\
& biologique (VS et CRP) \\
& Nombre élevé d'articulations gonflées \\
& - Présence de facteur rhumatoïde et d'ACPA, \\
& particulièrement en cas de taux élevés ( $\geq 3 \mathrm{~N}$ ) \\
- & Activité de la maladie modérée à forte malgré un \\
& traitement par csDMARD* \\
& - Echec de $\geq 2$ csDMARDs* \\
Rémission (selon des critères composites comprenant \\
une évaluation articulaire) maintenue sur une période \\
d'au moins 6 mois
\end{tabular}

ACPA: anticorps anti-peptides citrullinés; CRP: protéine C-réactive; VS: vitesse de sédimentation. * Facteurs de mauvaise réponse plutôt que de mauvais pronostic 
Tableau 2. Les recommandations 2018 de la Société française de rhumatologie pour la prise en charge de la polyarthrite rhumatoïde

\begin{tabular}{|c|c|c|c|c|}
\hline Principes généraux et recommandations & N.d.p & Grade & $\begin{array}{l}\text { Accord du } \\
\text { groupe de } \\
\text { travail } \\
\text { Moy.(DS) } \\
\end{array}$ & $\begin{array}{l}\text { Accord } \\
\text { du groupe } \\
\text { de lecture } \\
\text { Moy.(DS) }\end{array}$ \\
\hline \multicolumn{5}{|l|}{ PRINCIPES GENERAUX } \\
\hline $\begin{array}{l}\text { A. La prise en charge des patients atteints de PR } \\
\text { nécessite une concertation entre le rhumatologue } \\
\text { et le patient, dans le cadre d'une décision } \\
\text { médicale partagée reposant sur l'information et } \\
\text { l'éducation du patient. }\end{array}$ & NA & NA & $10(0)$ & $9,3(1,2)$ \\
\hline $\begin{array}{l}\text { B. Le rhumatologue est le spécialiste qui doit } \\
\text { prendre en charge les patients atteints de PR. Le } \\
\text { médecin généraliste joue un rôle important pour } \\
\text { la détection de la maladie et, en coordination } \\
\text { avec le rhumatologue, pour le suivi du patient. }\end{array}$ & NA & NA & $9,7(0,8)$ & $9,8(0,6)$ \\
\hline $\begin{array}{l}\text { C. Tout patient atteint de PR devrait bénéficier } \\
\text { d'une prise en charge globale incluant les } \\
\text { traitements médicamenteux, des mesures } \\
\text { d'éducation thérapeutique et, selon les cas, une } \\
\text { prise en charge des comorbidités, un soutien } \\
\text { psychologique, un accompagnement } \\
\text { socioprofessionnel, une rééducation } \\
\text { fonctionnelle ou le recours à la chirurgie. }\end{array}$ & NA & NA & $9,7(0,6)$ & $9,2(1,3)$ \\
\hline $\begin{array}{l}\text { D. Le coût de la PR, de ses conséquences et de } \\
\text { ses traitements, à l'échelon individuel et sociétal, } \\
\text { devrait être pris en considération dans les } \\
\text { orientations thérapeutiques. }\end{array}$ & NA & NA & $9,3(1,0)$ & $7,6(2,9)$ \\
\hline \multicolumn{5}{|l|}{ RECOMMANDATIONS } \\
\hline \multicolumn{5}{|l|}{ Diagnostic } \\
\hline $\begin{array}{l}\text { 1. Le diagnostic de PR doit être: } \\
\text { - évoqué devant certains signes cliniques tels } \\
\text { qu'un gonflement articulaire (arthrite } \\
\text { clinique), une raideur matinale de plus de } 30 \\
\text { min, une douleur à la pression transverse des } \\
\text { mains ou des avants-pieds. } \\
\text { confirmé le plus tôt possible (idéalement } \\
\text { dans les } 6 \text { semaines) par l'examen clinique } \\
\text { du rhumatologue et par des examens } \\
\text { biologiques (vitesse de sédimentation, C- } \\
\text { réactive protéine, anticorps anti-peptides } \\
\text { citrullinés (ACPA), facteurs rhumatoïdes) et }\end{array}$ & $\mathrm{IIb}$ & $\mathrm{B}$ & $9,7(0,5)$ & $9,4(1,0)$ \\
\hline
\end{tabular}


d'imagerie (radiographies +/- échographie), après avoir éliminé les diagnostics différentiels

2. Dès que le diagnostic de PR est posé, un Ia traitement de fond doit être débuté.

3. L'objectif du traitement est d'atteindre et de maintenir la rémission clinique ou au minimum la faible activité. La rémission clinique est définie par l'absence de signes et symptômes d'activité inflammatoire significative.

4. L'activité de la maladie doit être mesurée par des critères composites validés, incluant les indices articulaires.

5. Le suivi de la maladie par le rhumatologue doit être fréquent (1 à 3 mois) tant que la maladie est active. S'il n'y a pas d'amélioration dans les 3 mois suivant le début du traitement ou si l'objectif thérapeutique n'a pas été atteint à 6 mois, le traitement doit être ajusté.

$\begin{array}{lll}\text { A } & 9,9(0,5) & 9,4(1,0) \\ \text { A } & 9,8(0,4) & 9,3(1,1)\end{array}$

Ia

B

$9,6(0,7)$

IIb $\quad$ B $\quad 9,8(0,4) \quad 9,3(1,2)$

\section{Traitement de $1^{\mathrm{e}}$ ligne}

6. Le méthotrexate est le traitement de fond de Ia

A

$9,3(1,0)$

$8,5(2,0)$

première ligne chez les patients ayant une PR active avec une posologie initiale d'au moins 10 $\mathrm{mg} / \mathrm{semaine}$ et une posologie optimale atteinte au maximum en 4 à 8 semaines.

7. Chez les patients naïfs de traitement de fond, Ia A $\quad 9,5(0,8)$ en cas de contre-indication au méthotrexate ou d'intolérance précoce, le leflunomide ou la sulfasalazine sont une alternative thérapeutique.

8. Dans l'attente de l'efficacité d'un traitement de fond synthétique conventionnel, une Ia B $9,0(1,2)$ corticothérapie orale ou injectable peut être proposée en respectant une dose cumulée faible, si possible sur une période maximale de 6 mois. La corticothérapie sera diminuée et arrêtée aussi rapidement que possible.

\section{Traitement de $2^{\text {nde }}$ ligne et plus}

9. Chez les patients insuffisamment répondeurs ou intolérants au MTX, le traitement doit être optimisé.

- En présence de facteurs de mauvais \# $\mathrm{Ib} \quad$ \# A pronostic, l'addition d'une thérapeutique ciblée (biologique ou synthétique) peut être proposée (anti-TNF, abatacept, inhibiteur de 


\begin{tabular}{|c|c|c|c|c|}
\hline $\begin{array}{l}\text { la voie de l'IL6, inhibiteurs de JAK et dans } \\
\text { certaines circonstances rituximab) }{ }^{\#} \text {. } \\
\text { - En l'absence de facteurs de mauvais } \\
\text { pronostic, une rotation pour un autre } \\
\text { traitement de fond synthétique conventionnel } \\
\text { (LEF, SLZ) ou bien une association de } \\
\text { traitement de fond synthétiques } \\
\text { conventionnels peuvent être proposées }{ }^{\S} \text {. En } \\
\text { cas d'échec (ou de contre-indication), une } \\
\text { thérapeutique ciblée (biologique ou } \\
\text { synthétique) doit être envisagée. }\end{array}$ & ${ }^{\S} \mathrm{V}$ & ${ }^{\S} \mathrm{D}$ & & \\
\hline $\begin{array}{l}\text { 10. Toutes les thérapeutiques ciblées } \\
\text { (biologiques }^{*} \text { ou synthétiques }{ }^{\sharp} \text { ) doivent être } \\
\text { utilisées préférentiellement en association avec } \\
\text { le MTX. }\end{array}$ & $\begin{array}{l}{ }^{*} \mathrm{Ia} \\
{ }^{\#} \mathrm{Ib}\end{array}$ & A & $9,5(0,7)$ & $8,9(2,0)$ \\
\hline $\begin{array}{l}\text { 11. Les patients en échec d'une première } \\
\text { thérapeutique ciblée (biologique }{ }^{*} \text { ou } \\
\text { synthétique }^{\S} \text { ) doivent être traités par une autre } \\
\text { thérapeutique ciblée. En cas d'échec primaire, un } \\
\text { changement de mode d'action peut être } \\
\text { privilégié. }\end{array}$ & $\begin{array}{l}{ }^{*} \mathrm{Ia} \\
{ }^{\S} \mathrm{V}\end{array}$ & A & $9,6(0,6)$ & $9,0(1,5)$ \\
\hline \multicolumn{5}{|l|}{ Gestion de la rémission } \\
\hline $\begin{array}{l}\text { 12. En cas de rémission persistante et sans } \\
\text { corticoïdes, une décroissance de la thérapeutique } \\
\text { ciblée (biologique ou synthétique) doit être } \\
\text { envisagée. }\end{array}$ & $\mathrm{IIb}$ & $\mathrm{B}$ & $9,1(1,4)$ & $8,7(1,9)$ \\
\hline $\begin{array}{l}\text { 13. En cas de rémission persistante prolongée } \\
\text { sans thérapeutique ciblée et sans corticoïdes, une } \\
\text { réduction progressive des traitements de fond } \\
\text { conventionnels synthétiques peut être envisagée. }\end{array}$ & IV & $\mathrm{C}$ & $9,0(1,4)$ & $8,7(2,1)$ \\
\hline \multicolumn{5}{|l|}{ Prise en charge globale } \\
\hline $\begin{array}{l}\text { 14. Le choix et l'adaptation thérapeutiques } \\
\text { doivent intégrer d'autres facteurs que la mesure } \\
\text { de l'activité de la maladie comme la progression } \\
\text { structurale }{ }^{*} \text {, les maladies associées, la tolérance } \\
\text { des traitements, l'adhésion et l'avis du patient". }\end{array}$ & $\begin{array}{l}{ }^{*} \mathrm{Ia} \\
{ }^{\#} \mathrm{Ib}\end{array}$ & $\mathrm{C}$ & $9,8(0,4)$ & $9,6(0,8)$ \\
\hline $\begin{array}{l}\text { 15. Un dépistage et une évaluation périodique } \\
\text { des comorbidités, de leurs facteurs de risque et } \\
\text { de leur prise en charge doivent être réalisés\$. La } \\
\text { prise en charge doit être associée à des conseils } \\
\text { d'hygiène de vie (activité physique régulière, } \\
\text { arrêt du tabac, alimentation équilibrée, etc.) et la } \\
\text { mise à jour des vaccinations \& }\end{array}$ & $\begin{array}{l}{ }^{\$} \mathrm{IIb} \\
{ }^{\&} \mathrm{IV}\end{array}$ & $\mathrm{C}$ & $9,4(0,9)$ & $9,1(1,7)$ \\
\hline
\end{tabular}


Le grade des recommandations reflète le niveau de preuve (N.d.p): A correspond au niveau de preuve le plus élevé. NA : non applicable. ${ }^{\mathrm{a}}$ Accord noté entre 0 et 10 où 10 représente un accord complet 